\title{
On synonymy of linyphiid spiders of the Russian fauna. 3 (Arachnida: Aranei: Linyphiidae)
}

\author{
О синонимии пауков-Аинифиид фауны России. 3 \\ (Arachnida: Aranei: Linyphiidae)
}

\author{
Andrei V. Tanasevitch \\ A.В. Танасевич \\ Institute of Ecology and Evolution, Russian Academy of Sciences, Leninsky Prospect, 33, Moscow 119071, Russia. E-mail: \\ tanasevitch@gmail.com \\ Институт проблем экологии и эволюции РАН, Ленинский проспект, 33, Москва 119071, Россия.
}

KEY WORDS: Spiders, Linyphiidae, new synonyms, new combinations, new records.

КЛЮЧЕВЫЕ СЛОВА: Пауки, Linyphiidae, новые синонимы, новые комбинации, новые находки.

ABSTRACT. The following new synonyms are established: Alioranus avanturus Andreeva et Tyschchenko, 1970, syn.n. = A. chiardolae (di Caporiacco, 1935), comb.n. ex Gongylidiellum Simon, 1884; Gnathonarium sibirianum (Keyserling, 1886), syn.n. and G. columbianum (Emerton, 1923), syn.n. = G. taczanowskii (O. Pickard-Cambridge, 1873), non $G$. suppositum (Kulczyński, 1885); Pelecopsis steppensis Gnelitsa, 2008, syn.n. $=$ P. laptevi Tanasevitch et Fet, 1986; Porrhomma kulczynskii Staręga, 1974, syn.n. $=P$. boreale (Banks, 1899). The synonymy of G. famelicum (Keyserling, 1886) with G. suppositum (Kulczyński, 1885) is reaffirmed. Abundant material of $G$. dentatum (Wider, 1834) is revised, some of the samples from the Russian Far East being actually referred to G. suppositum, but some others, from Kamchatka (the Geysers Valley), as well as from the continental parts of the Russian Far East and central Siberia, which combine the characters of both G. dentatum and $G$. suppositum, are probably hybrids between these similar taxa. All records of Bathyphantes similis Kulczyński, 1894 from Russia are misidentifications and actually refer to B. gracilis (Blackwall, 1841). All Palaearctic records of Wabasso quaestio (Chamberlin, 1949) appear to represent $W$. replicatus (Holm, 1950). Tapinocyba incerta Kulczyński, 1916 , recorded by Tullgren [1955] in Sweden, actually refers to Tapinocyboides pygmaeus (Menge, 1869), thus the distributional pattern of this species, now Sibirocyba incerta, can be regarded as Siberian. Agyneta birulaioides Wunderlich, 1995 is being reported from Europe for the first time, due to its record in the steppe habitats of Kherson Area, Ukraine. Mecynargus minutipalpis Gnelitsa, 2011, from the Kalmyk Republic, and Tibioploides stigmosus (Xia et al., 2001), from the Kurile Islands, are new to the Russian fauna. Mecynargus longus (Kulczyński, 1882) is removed from the Russian list.
РЕЗЮМЕ. Установлена новая синонимия: Alioranus avanturus Andreeva et Tyschchenko, 1970, syn.n. $=$ A. chiardolae (di Caporiacco, 1935), comb.n. ex Gongylidiellum Simon, 1884; Gnathonarium sibirianum (Keyserling, 1886), syn.n. и G. columbianum (Emerton, 1923), syn.n. = G. taczanowskii (O. PickardCambridge, 1873), a не G. suppositum (Kulczyński, 1885); Pelecopsis steppensis Gnelitsa, 2008, syn.n.= P. laptevi Tanasevitch et Fet, 1986; Porrhomma kulczynskii Staręa, 1974, syn.n. = P. boreale (Banks, 1899). Подтверждена синонимия G. famelicum (Keyserling, 1886) и G. suppositum (Kulczyński, 1885). Пересмотрен материал по G. dentatum (Wider, 1834): установлено, что некоторые экземпляры из российского Дальнего Востока на самом деле являются $G$. suppositum, а экземпляры из Долины гейзеров (Камчатка), а также из континентальных регионов российского Дальнего Востока и Центральной Сибири несут признаки как G. dentatum, так и G. suppositum: вероятно, эта территория является ареной гибридизации двух близких таксонов. Все находки Bathyphantes similis Kulczyński, 1894 в России являются ошибочными и относятся к B. gracilis (Blackwall, 1841). Все находки Wabasso quaestio (Chamberlin, 1949) в Палеарктике относятся к $W$. replicatus (Holm, 1950). Tapinocyba incerta Kulczyński, 1916, отмеченный в Швеции [Tullgren, 1955], в действительности является Tapinocyboides pygmaeus (Menge, 1869), т.о., ареал этого вида, ныне Sibirocyba incerta, следует рассматривать как сибирский. Agyneta birulaioides Wunderlich, 1995 впервые найден в Европе (степи Херсонщины, Украина). Mecynargus minutipalpis Gnelitsa, 2011 найденный в Калмыкии и Tibioploides stigmosus (Xia et al., 2001) с Курильских островов - новые для фауны России; напротив, Mecynargus longus (Kulczyński, 1882) выведен из ее состава. 


\section{Introduction}

This paper is the third in a series [see Tanasevitch, 2010,2011 ] of contributions reassessing the validity and status of certain linyphiid species from the Russian fauna sensu lato, i.e., considered in the scope of the former Soviet Union. Besides this, it corrects some previous misidentifications, revises several identities, as well as puts on record some new or interesting findings of linyphiids.

\section{Material and Methods}

This paper is mainly based on the spider material kept at the Zoological Museum of the Moscow State University, Moscow, Russia, as well as in the personal collections of Andrei Tanasevitch, Moscow, Alexander Ponomarev, Rostov-on-Don, Russia, and Donald Buckle, Saskatchewan, Canada. Some types and comparative material have been obtained from Institute for Biological Problems of the North, Magadan, Russia; Muséum d'Histoire naturelle, Geneva, Switzerland; Museo Civico di Storia naturale, Milano, Italy; Naturhistoriska Riksmuseet, Stockholm, Sweden; Perm State University, Perm, Russia; Senckenberg Museum, Frankfurt am Main, Germany; Department of Zoology, University of Tel Aviv, Israel; University of Alaska Museum, Fairbanks, USA; Zoological Institute, Russian Academy of Sciences, St. Petersburg, Russia; Museum and Institute of Zoology, Warsaw, Poland, and Zoological Museum, Turku University, Turku, Finland.

Drawings are based on photographs taken by a digital camera.

Abbreviations used in the text and figures: CAP personal collection of A. Ponomarev; CAT — personal collection of A. Tanasevitch; CDB - personal collection of D. Buckle; CVS - collection of Sytschevskaya (ZMMU); IBPN — Institute for Biological Problems of the North, Magadan; Ma - mastidion; MCSNM - Museo civico di Storia naturale, Milano; MHNG — Muséum d'Histoire naturelle, Geneva; MIZ — Zoological Museum of the Institute of Zoology, Warsaw; NHMF — Natural History Museum University of Florence; NHRS Naturhistoriska Riksmuseet, Stockholm; N.R. — Nature Reserve; PSU — Department of Zoology, Perm State University, Perm; SMF — Senckenberg Museum, Frankfurt am Main; UAM - University of Alaska Museum, Fairbanks; UTA - Department of Zoology, University of Tel Aviv; ZIN - Zoological Institute, Russian Academy of Science, St. Petersburg; ZMMU - Zoological Museum, Moscow State University, Moscow; ZMTU Zoological Museum, Turku University, Turku.

\section{Results}

Alioranus chiardolae (di Caporiacco, 1935), comb.n.

1935 Gongylidiellum chiardolae di Caporiacco: 169, P1. III, fig. $1(\mathrm{a}-\mathrm{c}), \sigma^{7} \&$ ㅇ, MCSNM, type examined.
1970 Alioranus avanturus Andreeva et Tyschchenko: 38, fig. 1 (a-ж), $\sigma^{\top} \&$ \& , syn.n.

1976 A. avanturus. - ANDREEVA: 61, figs 62-68, o7 \&

1989 A. avanturus. - Tanasevitch: 124, figs 110-114, o \& $\&$.

TYPE MATERIAL EXAMINED. Gongylidiellum chiardolai: $2 \sigma^{\top} \sigma^{T}, 4$ 90 ; $1 \sigma^{T}$ designated herewith as lectotype (MCSNM, \#137), Karakorum, Dumultar, Punmah, 3950 m a.s.1., 5.VIII.1929; 1 ( (MCSNM, \#165), Karakorum, Fra Lamidukha e Punmah, 3600 m a.s.1., 8.VIII.1929; 3 ○ $\sigma^{7}$ (MCSNM, \#127b): Bardumal, $3300 \mathrm{~m}$ a.s.1., 2.VIII.1929; 1 ○', 1 क (MCSNM w/o \#), Bardumal, 3300 m a.s.1., 2.VIII.1929.

MATERIAL EXAMINED. Alioranus avanturus: $4 \sigma^{7} \sigma^{7}, 18$ १ᄋ (ZMMU), UZBEKISTAN, Pamir-Alay Mts, Surkhandarya Area, Kugitang Mt. Ridge, Baglydara Valley, 1600-1800 m a.s.1., Juniperus sparse forest, litter along spring, 25-27.V.1985, leg. A. Ta-

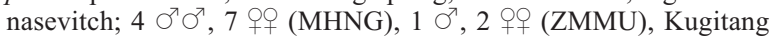
Mt. Ridge, near Kampyrtypa (= Kempir-Tyube), in grass near spring, $1800 \mathrm{~m}$ a.s.1., 19.V.1985; 2 ๙ $^{\top}, 1$ o 1 (ZMMU), same, under stones in spring channel, 15.V.1985, all leg. A. Tanasevitch; $2 \sigma^{7} \sigma^{7}$ (MHNG), 10 Oᄋ (ZMMU), TURKMENISTAN, Pamir-Alay Mts, Kugitang-Tau Mt. Ridge, near Khodjapil-Ata, 1200 m a.s.1., valley, under stones along spring, 10.V.1985, leg. A. Tanasevitch; $2 \sigma^{\top} \sigma^{7}, 1$ (ZMMU), same, valley, under stones on slope, $1200 \mathrm{~m}$ a.s.1., 10.V.1985; 1 (ZMMU), KYRGYZSTAN, North-Eastern Tian Shan Mts, Ysyk-Kol [= Issyk-Kul] Area, Terskey Ala-Too Mt. Ridge, Koilyu Valley, $2800 \mathrm{~m}$ a.s.1., Picea forest, 16.VII.1983, leg. S. Ovtchinnikov; $1 \sigma^{7}, 1$ (ZMMU), Northern Tian Shan Mts, Ysyk-Kol [= Issyk-Kul] Area, Terskey Ala-Too Mt. Ridge, ChonUryukty Valley, 2100 m a.s.l., 11.VIII.1984, leg. S. Ovtchinnikov; $2 \sigma^{\top} \sigma^{\top}$ (CAT), South-Western Tian Shan Mts, Jalal-Abad [= DzhalalAbad] Area, Nooken [= Lenin] District, W foothills of Chaaktau Mt. Ridge, ca $1 \mathrm{~km} \mathrm{SE}$ of Tashkomur [= Tash-Kumyr], SaryKamysh-Sai River Valley, ca $41^{\circ} 20^{\prime} 00.0^{\prime \prime} \mathrm{N}, 72^{\circ} 13^{\prime} 30.0^{\prime \prime} \mathrm{E}, 600$ $650 \mathrm{~m}$ a.s.1., 13-25.VIII.1985, leg. D. Logunov; 1 ๆ', 2 우 (CAT), Inner Tian Shan Mts Naryn Area, Naryn [= Tian Shan] District, N slope of Naryn-Too Mt. Ridge, Iyri-Suu [= Iiri-Suu Southern] River Canyon, ca $3.5 \mathrm{~km}$ E of Tash-Bashat, ca $41^{\circ} 28^{\prime} 01.0^{\prime \prime} \mathrm{N}$, $76^{\circ} 27^{\prime} 03.4^{\prime \prime} \mathrm{E}, 2400-2450 \mathrm{~m}$ a.s.1., 19.VII.1987, leg. S. Ovtchinnikov; 1 \& (ZMMU), TAJIKISTAN, W-Pamir Mts, Gorno-Badakhshan Autonomous Region, Shugnanskiy Mt. Ridge, near Khorog, 2000-3000 m a.s.1., 17.V.1970, leg. E. Andreeva.

REMARKS. Gongylidiellum chiardolae was originally described from both sexes from Karakorum (3300$3600 \mathrm{~m}$ a.s.1.) by di Caporiacco [1935]. The description was accompanied by such poor line drawings that no identification could be made without a restudy of type material.

Alioranus avanturus Andreeva et Tyschchenko, 1970 was based on a male from Varzob Valley, Pamir-Alay Mts, and two females from the "Tigrovaya Balka" Nature Rederve, both localities in Tajikistan [Andreeva \& Tyschchenko, 1970]. Later, A. avanturus was recorded from different parts of Central Asia: Kugitang Mt. Ridge, Pamir-Alay Mts, 1400-1800 m a.s.l. (Uzbekistan and Turkmenistan); from Terskey Ala-Too Mt. Ridge, Tian Shan Mts, 2100-2800 m a.s.l. (Kyrgyzstan), and from Pamir Mts, Gorno-Badakhshan Autonomous Region, 2000-3000 m a.s.l. (Tajikistan), all records by Tanasevitch [1989]. A study of the types of $G$. chiardolae shows its complete identity to $A$. avanturus. Thus, the latter species in a new subjective junior synonym of $A$. chiardolae, syn.n. \& comb.n.

DISTRIBUTION. The mountains of Central Asia: Tian Shan, Pamir-Alay, Pamir, Himalaya (Karakorum); occurring from 300 up to $3000 \mathrm{~m}$ a.s.l.

PATTERN. Central Asian. 
Gnathonarium suppositum (Kulczyński, 1885) Figs $1-6 ; 13-21 ; 28-37$.

1885 Gongylidium suppositum Kulczyński: 36.

1886 Erigone famelica Keyserling: 186, pl. XVII: 246, ○', NMNH, type examined, syn. confirm.

1886 E. sibiriana Keyserling: 189, pl. XVII, fig. 249,, , NMNH, type examined, syn. confirm.

1935 Gnathonarium dentatum. - Sytschevskaya: 91, misidentification, examined.

1960 G. famelicum. - Holm: 117, fig. 9, ㅇ.

1992 G. dentatum. - Eskov: 55, in part, misidentification, examined.

1993 G. dentatum. - Marusik et al.: 76, misidentification, examined.

2007 G. dentatum. - Tanasevitch: 256, misidentification, examined.

2007 G. dentatum. - Marusik et al.: 46, possible misidentification.

For more information on findings and misidentifications see Eskov [1994].

TYPE MATERIAL EXAMINED. Erigone famelica Keyserling, 1886: $1 \sigma^{7}$, type, U.S. National Museum Collection NMNH, Washington, \#1638. Erigone sibiriana Keyserling, 1886: 1 + , type, U.S. National Museum Collection NMNH, Washington, \#1652.

COMPARATIVE MATERIAL EXAMINED. Gnathonarium suppositum: $1 \sigma^{7}, 1$ क (UAM), recognized by photos, USA, Alaska State, Aleutian Islands, Agattu Isl., $15 \mathrm{~m}$ a.s.1., $52^{\circ} 28.385^{\prime} \mathrm{N}$ $173^{\circ} 35.439^{\prime}$ E, beach, 5-19.VI.2008, leg. L. Kenney \& R. Kaler; 4 우 (ZMMU, CVS, Ta-2806, as G. dentatum), RUSSIA, Kamchatka Peninsula, Ust'-Kamchatsk, in a tent, 20-24.IX.1930, leg. V. Sytschevskaya; $1 \sigma^{7}$ (ZMMU, CVS, Ta-2808, as G. dentatum), same, Ust'-Kamchatsk, Lake Nerpech'ye, XI.1932, leg. V. Sytschevskaya; 1 ㅇ, (ZMMU, CVS, Ta-2805, as G. dentatum), Kamchatka, Kamchatkan River near Ust'-Kamchatsk, in grass, 3-4.VIII.1930, leg. V. Sytschevskaya; 1 , (ZMMU, as G. famelicum), Commander Islands, Mednyi Island, Cape Drovenskoy, grassy tundra, under twigs and branches, 8.VII.1983, leg. A. Zimenko; 1 (ZMMU, as G. famelicum), Mednyi Island, VII-VIII.1978, leg. A. Panteleev; 4 + ,,$(\mathrm{CAT}$, as G. dentatum) Simushir Island, Brouton Bay area, $47.141086^{\circ} \mathrm{N}, 152.266331^{\circ} \mathrm{E}$, shrubby slopes on coast of Brouton Bay with Alnus sp., Betula sp., Pinus pumila, in litter and under stones, 15-16.VIII.2002, leg. A. Tanasevitch; $4 \sigma^{\top} \sigma^{\top}, 17$ 우, (CAT, as $G$. dentatum), environs of Brouton Bay, $47.111881^{\circ} \mathrm{N}$, $152.190622^{\circ} \mathrm{E}$, top of ridge, $300-350 \mathrm{~m}$ a.s.1., lichen-moss tundra with shrubs of Pinus pumila; Empetrum sp., Arctous sp., 1516.VIII.2002, leg. A. Tanasevitch; $1{ }^{7}, 2$ (ZMMU, as $G$. dentatum), Sakhalin, Makarov District, environs of Pugachevo, 5 6.IX.1983, leg. A. Basarukin; $5 \sigma^{7} \sigma^{7}, 12$ +o (ZMMU, as G. dentatum), Tomarino District, Lake Ainskoye, Ptich'ya River, wet meadow, 24-25.V.1984, leg. A. Basarukin; 1 , (ZMMU, as $G$. dentatum), Dolinsk District, Pokrovka, 18-23.VII.1987, leg. A. Basarukin; 2 우, (ZMMU, as G. dentatum), Maritime Prov. (= Primorskiy Krai) Vladivostok, Artyom, 3.VI.1981, leg. P. Dunin.

REMARKS. Gnathonarium suppositum was originally described from a single female from the environs of Petropavlovsk-Kamchatskiy, Kamchatka Peninsula [Kulczyński, 1885], in Gongylidium Karsch, 1881. The holotype is probably lost, having been found neither in MIZ, Warsaw (Dominika Mierzwa-Szymkowiak, pers. comm.) nor in ZIN, St. Petersburg (Kirill Mikhailov, pers. comm.).

Eskov [1988: 105], without any explanation, synonymized both G. columbianum Emerton, 1923, and G. famelicum (Keyserling, 1886) under G. suppositum. Also groundlessly, Eskov \& Marusik [1994: 67] synonymized G. sibirianum (Keyserling, 1886) with $G$. suppositum (Kulczyński, 1885).
However, this becoming clear only now, the synonymy G. columbianum $=G$. suppositum is wrong (see remarks under G. taczanowskii). In contrast, the synonymy $G$. suppositum $=$ G. famelicum and G. sibirianum $=G$. suppositum are correct. Even though the holotype of G. suppositum could not be relocated, examination of the type of $G$. famelicum, described from Sitka, Alaska [Keyserling, 1886], and its comparison with G. suppositum material from the Aleutian Islands, USA, Kamchatka and the other places in the Russian Far East referred to above show that both these species are conspecific (syn. confirm.). An examination of the type of G. sibirianum, which was originally described from a female from the Commander Islands, Russia [Keyserling, 1886], shows that this species is also conspecific to G. suppositum (syn. confirm.)

The differences between G. suppositum and G. dentatum (Wider, 1834) are small and often overlapping. Thus, the carapace of the male of G. suppositum is characterized by a slight and regular curve of its back. In addition, this being even more marked, a much smaller mastidion is only poorly visible from behind the chelicera in lateral view. The tip of the male palpal tibia is narrowed at its base and bent down a bit more evidently than in G. dentatum. The epigyne in G. suppositum differs by the loops of the entrance ducts slightly sunken caudad, as well as by the slightly larger "pocket" on the epigynal median plate.

NOTE. Gnathonarium samples collected from the Geysers Valley in Kamchatka combine the characters both of G. suppositum and G. dentatum, probably being their hybrids (see below under $G$. dentatum).

DISTRIBUTION: Kamchatka and islands in the Russian Far East: Sakhalin, Kurile Islands, Commander Islands; USA and Canada: Aleutian Islands, Alaska to Manitoba in the east.

\section{Gnathonarium dentatum (Wider, 1834)}

Figs 7-12, 22-27, 38-94.

$$
\begin{aligned}
& 1992 \text { G. dentatum. - Eskov \& Marusik: 71, examined. } \\
& 1992 \text { G. dentatum. - Eskov: 77, examined. } \\
& 2004 \text { G. dentatum. - Tanasevitch \& Trilikauskas: 81, exam- } \\
& 2006 \text { G. dentatum. - Tanasevitch: 358, examined. } \\
& 2006 \text { G. dentatum. - Trilikauskas \& Tanasevitch: 341, exam- } \\
& 2008 \text { G. dentatum. - Tanasevitch: 123, examined. } \\
& \text { MATERIAL EXAMINED. } 1 \sigma^{7} \text { (ZMMU), DENMARK, Ho- }
\end{aligned}
$$
ined. ined. bro, Fyrkat, 22.VII.2000, leg. K. Mikhailov; $5 \sigma^{7} \sigma^{7}, 6$ 오 (UTA), ISRAEL, south shore of Lake Kinneret near Deganya, 26.IV.2010, leg. C. Drees \& L. Friedman; $3 \sigma^{\top} \sigma^{7}$ (UTA), same, 30.I.2011, leg. C. Drees \& L. Friedman; 2 우 (UTA), same, 12.II.2010, leg. C. Drees \& L. Friedman; 1 (UTA), $6 \mathrm{~km} \mathrm{~S}$ of Ahusam, Pura $\left(31^{\circ} 29^{\prime} 49^{\prime \prime N} 34^{\circ} 46^{\prime} 50 " \mathrm{\prime}\right.$ ), 24.II.2011, leg. C. Drees \& L. Friedman; $7 \sigma^{\top} \sigma^{7}, 6$ 우 (UTA), $2 \mathrm{~km} \mathrm{~W}$ of Yeruham, bank of Lake

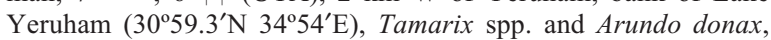
$450 \mathrm{~m}$ a.s.1., pitfall traps, 14.III.2011, leg. C. Drees \& L. Friedman; $3 \sigma^{7} \sigma^{7}$ (UTA), same, 3.VIII.2010, leg. C. Drees \& L. Friedman; 12 $\sigma^{\top} \sigma^{\top} \& 17$ OO (UTA), same, 7.III.2011, leg. C. Drees \& L. Friedman; $2 \sigma^{\top} \sigma^{\top}, 2$ 우 (UTA), Upper Galilee, env. of Lake Hula, 21.II.2011, leg. C. Drees \& L. Friedman; $10 \sigma^{\top} \sigma^{7}, 10$ 우 (UTA), same, 30.I.2011, leg. C. Drees \& L. Friedman; 1 \%", 6 우 (CAT), Caucasus, AZERBAIJAN, Lenkoran Distr., Girkanskiy (= Hyrcan) 


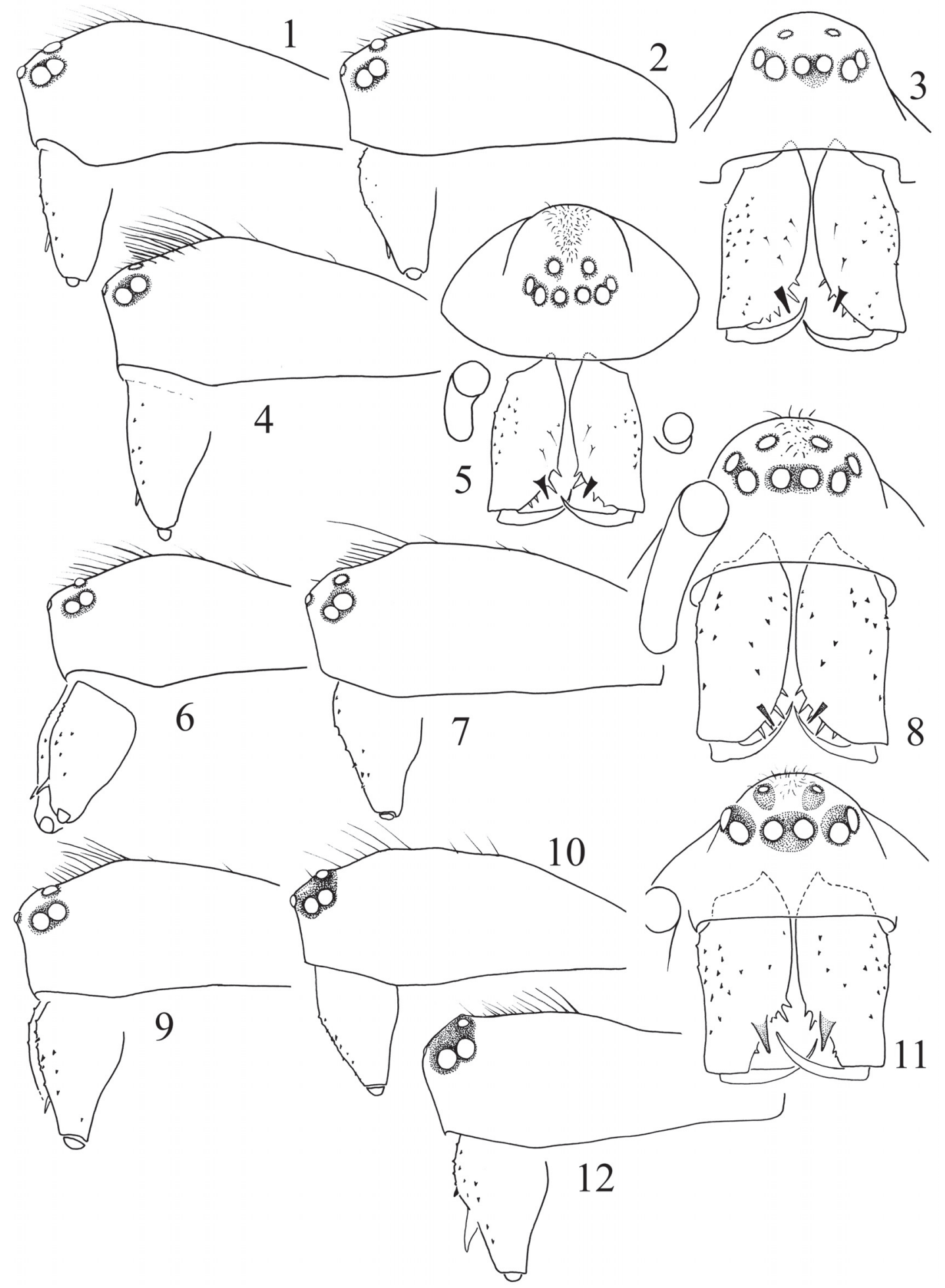

Figs 1-12. Male carapace of Gnathonarium famelicum (Keyserling, 1886), type (1), G. suppositum (Kulczyński, 1885) (2-6) \& G. aff. dentatum (Wider, 1834) (7-12): 1, 2, 4, 6, 7, 9, 10,12 - lateral view, 3, 5, 8, 11 - frontal view. 1 - type from Sitka, Alaska; 2 specimen from Dalnegorsk, Maritime Prov.; 3, 4 - specimen from Ptich'ya River, Sakhalin; 5, 6 - specimen from Simushir Island, Kurile Islands; 7-10 - specimens from Geysers Valley, Kamchatka; 11,12 - specimen from Norskiy N.R., Amurskaya Area. Not to scale.

Рис. 1-12. Карапакс самца Gnathonarium famelicum (Keyserling, 1886), тип (1), G. suppositum (Kulczyński, 1885) (2-6) \& G. aff. dentatum (Wider, 1834) (7-12): 1, 2, 4, 6, 7, 9, 10, 12 - вид сбоку, 3, 5, 8, 11 - вид спереди. 1 - тип из Ситки, Аляска; 2 экземпляр из Дальнегорска, Приморье; 3,4 - экземпляр с реки Птичья, Сахалин; 5, 6 - экземпляр с о-ва Симушир, Курильские ова; 7-10 - экземпляры из Долины гейзеров, Камчатка; 11, 12 - экземпляр из Норского заповедника, Амурская обл. Не в масштабе. 


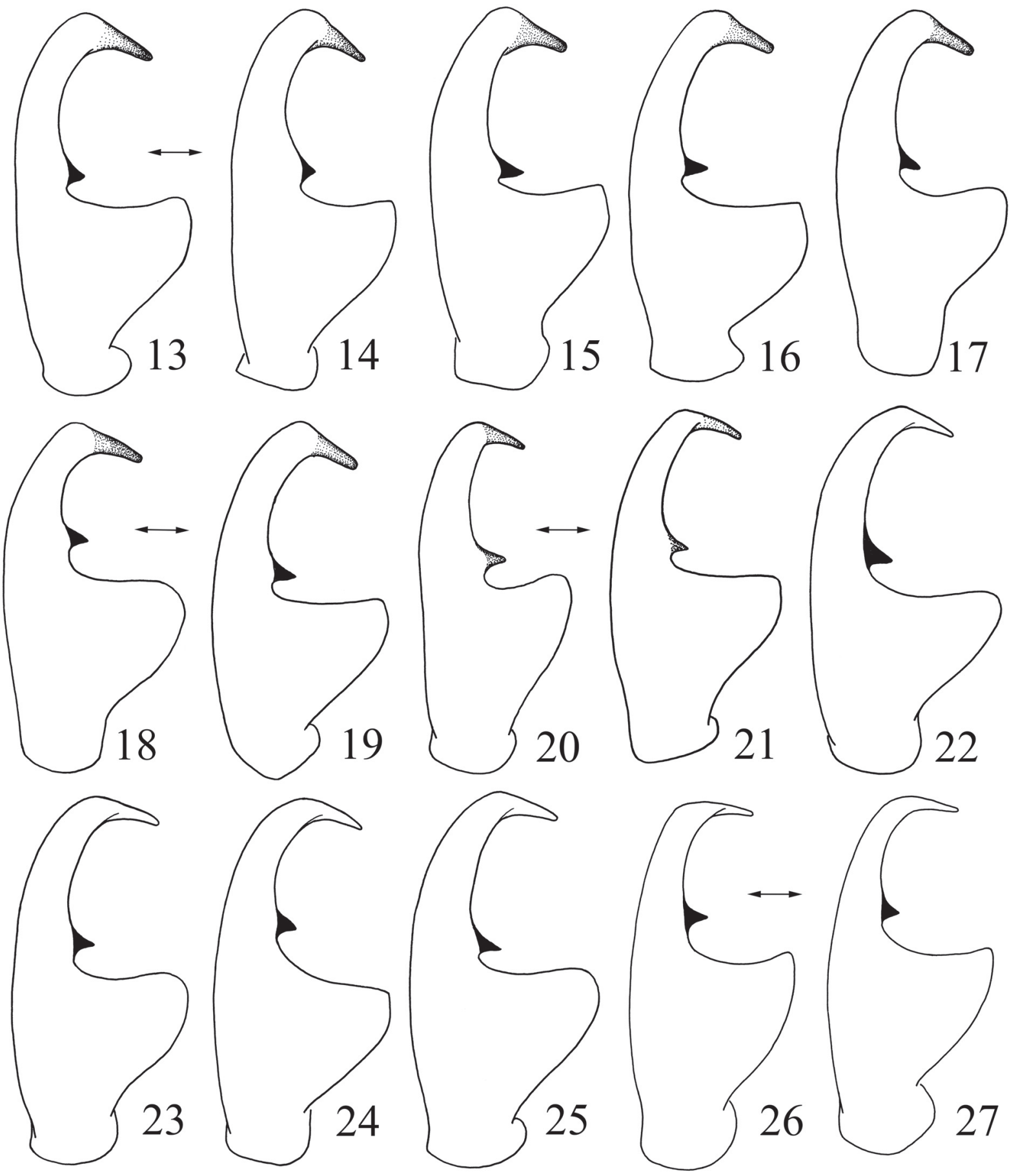

Figs 13-27. Male left palpal tibia of Gnathonarium famelicum (Keyserling, 1886), type (13, 14), G. suppositum (Kulczyński, 1885) (15-21) \& G. aff. dentatum (Wider, 1834) (22-27): 13, 14 - type from Sitka, Alaska; 15, 16 - specimens from Ptich'ya River, Sakhalin; 17 - specimen from Simushir Island, Kurile Islands; 18, 19 - specimen from Pugachevo, Sakhalin, different aspects; 20, 21 - specimen from Dalnegorsk, Maritime Prov., different aspects; 22-25 — specimens from Geysers Valley, Kamchatka; 26, 27 — specimen from Norskiy N.R., Amurskaya Area, different aspects. Arrows indicate which details belong to the same specimen. Not to scale.

Рис. 13-27. Голень левой пальпы самца Gnathonarium famelicum (Keyserling, 1886, тип $(13,14)$, G. suppositum (Kulczyński, 1885) (15-21) \& G. aff. dentatum (Wider, 1834) (22-27): 13, 14 - тип из Ситки, Аляска; 15,16 - экземпляр с реки Птичья, Сахалин; 17 - экземпляр с о-ва Симушир, Курильские о-ва; 18,19 - экземпляр из Пугачево, Сахалин, разный аспект; 20, 21 экземпляр из Дальнегорска, Приморье, разный аспект; 22-25 — экземпляры из Долины гейзеров, Камчатка; 26, 27 - экземпляр из Норского заповедника, Амурская обл., разный аспект. Стрелки указывают на один и тот же экземпляр. Не в масштабе. 

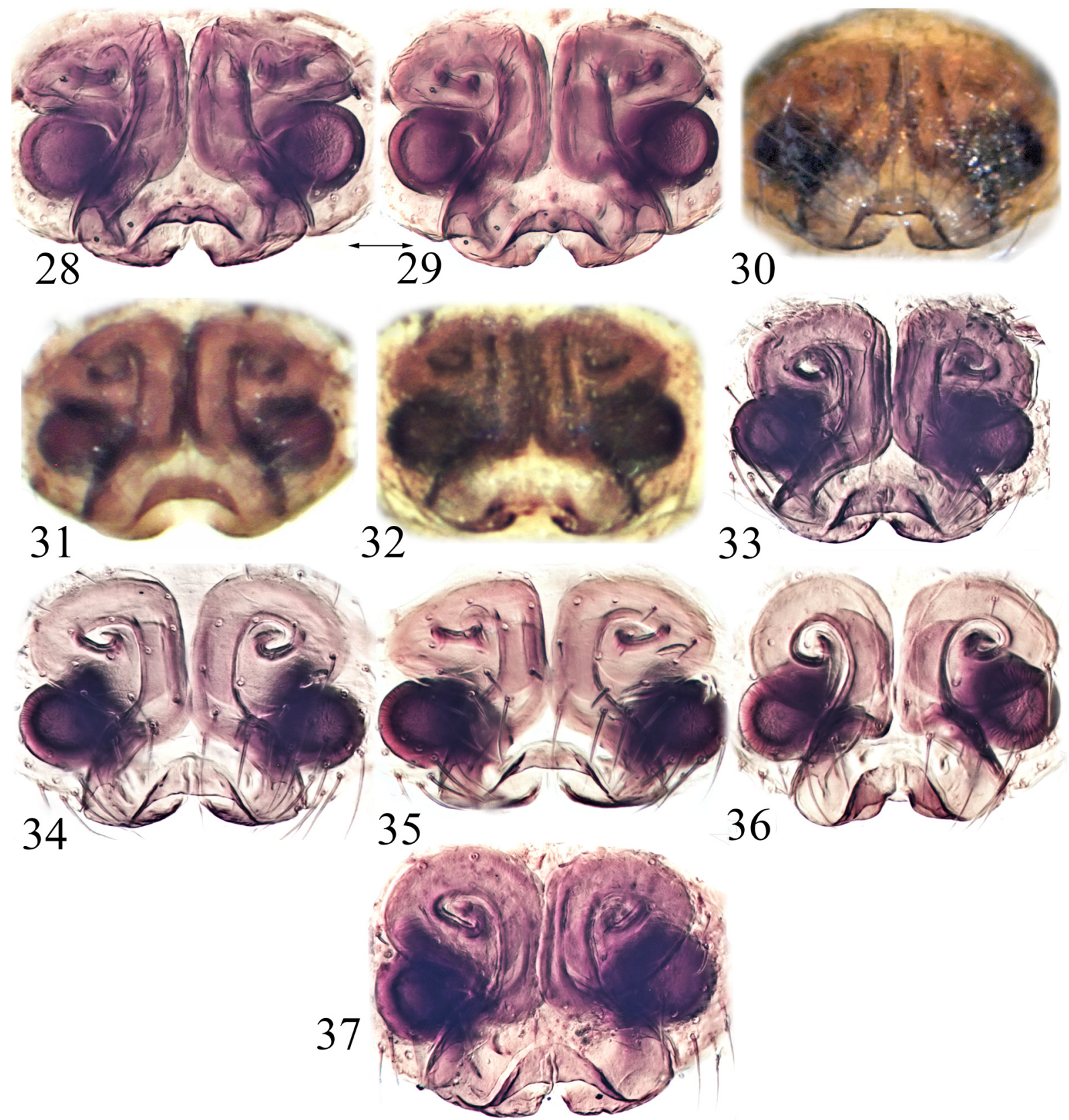

Figs 28-37. Epigyne (ventral view) of Gnathonarium sibirianum (Keyserling, 1886), type (28,29) \& G. suppositum (Kulczyński, 1885) (30-37): 28, 29 - type from Commander Islands, different aspects; 30 - specimen from Agattu Island, Aleutian Islands; 31,32 specimens from Simushir Island, Kurile Islands; 33 - specimen from Pugachevo, Sakhalin; 34-36 - specimens from Ptich'ya River, Sakhalin; 37 - specimen from Mednyi Island, Commander Islands. Arrows indicate which details belong to the same specimen. Not to scale.

Рис. 28-37. Эпигина (вид снизу) Gnathonarium sibirianum (Keyserling, 1886), тип (28, 29) \& G. suppositum (Kulczyński, 1885) (30-37): 28, 29 - тип с Командорских о-вов, разный аспект; 30 - экземпляр с о-ва Агатту, Алеутские о-ва; 31, 32 - экземпляры с о-ва Симушир, Курильские о-ва; 33 - экземпляр из Пугачево, Сахалин; 34-36 - экземпляры с реки Птичья, Сахалин; 37 экземпляр с о-ва Медный, Командорские о-ва. Стрелки указывают на один и тот же экземпляр. Не в масштабе.

N.R., near Alexeevka, Typha and Scirpus thickets, 2.VII.1983, leg. D. Logunov; 1 ( (CAT), Kirovsk, 200 m a.s.l., 8.V.1985, leg. P Dunin; $1 \mathrm{O}^{7}, 2$ 워 (CAT), Caucasus, GEORGIA, Poti, Paleostomi N.R., swamp, Alnus forest, litter, 12. IV. 1983, leg. S. Golovatch; 1 $\mathrm{O}^{7}$ (CAT), KAZAKHSTAN, Semipalatinsk Area, ca $8 \mathrm{~km} \mathrm{NW}$ of Semenovka, 5-9.VI.1998, leg. A. Gromov (new locality); 1 (CAT), KYRGYZSTAN, North Tien Shan, Issyk-Kul Area, flood- lands of Tyun River, Santash Valley, $3000 \mathrm{~m}$ a.s.l., steppe meadow, 23.VII.1983, leg. S. Ovtchinnikov; $70^{7} 0^{7}, 7$ 우 (CAT), West Tian Shan Mts, Osh Area, Fergansky Mt. Ridge, near Yarodar, $1500 \mathrm{~m}$ a.s.l., wet meadow, 10.V.1983, leg. A. Tanasevitch; 15 $\sigma^{7} \sigma^{7} \&$ 우오 (ZMMU), near Osh City, 10.I.1966, leg. unknown; $10^{7}$, 1 ( $($ ZMMU), UZBEKISTAN, Fergana [= Fargona] Area, Yaz'yavan [= Yozyovon] District, Karakalpak steppe, ca $13 \mathrm{~km} \mathrm{~W}$ of Yaz'yavan 


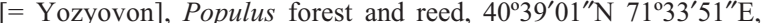
19.V.2002, leg. A. Gromov; $10^{7}, 2$ ㅇ (ZMMU), RUSSIA, Kostroma Area, Manturovo District, near Ugory, 23.VIII.1983, leg. E. Veselova; 5 O $^{7} 3$ 우 (CAT), Tyumen' Area, ca $50 \mathrm{~km} \mathrm{~N}$ of Tobolsk, "Missiya" Field Station, 3.VI.2004, leg. L. Rybalov; 8 $\sigma^{7} \sigma^{7}, 10$ 오 (CAT), Tyumen Area, Yuganskiy nature Reserve, summer, 2000-2003, leg. A. Ryvkin; 3 O $^{7}$ (ZMMU), Krasnoyarsk Prov., West Sayan, env. of Ermakovskoye, taiga, swamp, Carex, Scirpus, Sphagnum, 21.VII.1984, leg. A. Ryvkin;

G. aff. dentatum (Figs 7-12, 22-27, 49-51, 65-67, 80-94): 50 $0^{7} \sigma^{7} \&$ 우 (CAT, as G. dentatum), Kamchatka, Kronotskiy N.R., Geysers Valley, in grass near thermal sources, 30.VIII.1987, leg. A. Tanasevitch; 2 우 (CAT, as G. dentatum), Khabarovsk Prov., near Chegdomyn, VIII-IX, leg. L. Trilikauskas; 1 , (CAT), Verkhnebureinskiy District, Ust'-Urgal Nature Park, near Semicha River mouth, $280 \mathrm{~m}$ a.s.1., in oyster fungi on dead standing alder trunk, 27.VIII.2009, leg. A. Ryvkin; $1 O^{7}$ (CAT, as G. dentatum), Bureinskiy N.R., right side of Levaya Bureya River near mouth of Lan River, $610 \mathrm{~m}$ a.s.l., among Carex spp. at sandy-argillaceous bank of small puddle near edge of spit, 24.VIII.2010, leg. A. Ryvkin; 1 $\mathrm{O}^{7}$ (CAT, as G. dentatum), Amurskaya Area, near Belogorsk Tom' River bank, pasture and public beach, sweeping on sparse low grass and bushes, 6.VII.2005, leg. E. Veselova \& A. Ryvkin; $10^{7}$ (CAT, as G. dentatum), Tom' River bank near Belogorsk, pasture and public beach, sweeping on sparse low grass and, 6.VII.2005, leg. E. Veselova \& A. Ryvkin; $2 \bigcirc^{7} \sigma^{7}, 2$ 우 (CAT, as G. dentatum), Selemdzhinskiy District, Norskiy N.R., Nora River basin near Maltsevskiy cordon, $\mathrm{W}$ side of Maltsevskoye Lake, $210 \mathrm{~m}$ a.s.l., mosses and litter on swampy lakeside with tussocks of Carex spp., Poaceae gen. spp., Spiraea sp., Salix sp., 1.X.2008, leg. E. Veselova \& A Ryvkin; 2 O $^{7}, 2$ 우 (CAT), same, W side of Maltsevskoye Lake, $210 \mathrm{~m}$ a.s.l., mosses and litter on swampy lakeside with tussocks of Carex spp., Poaceae gen. spp., Spiraea sp., Salix sp., 1.X.2008, leg. E. Veselova \& A. Ryvkin; 1 (CAT, as G. dentatum), same, Burunda River, $1 \mathrm{~km}$ below Burunda Cordon, mosses (Plagiomnium sp. etc.) and leaf litter under Salix sp. with Poaceae, Carex sp., Filipendula palmata, Pyrola sp., 25.IX.2004, leg. A. Ryvkin; 1 + (CAT, as $G$. dentatum), Burunda River near Ozyornyi Rill mouth, 2.X.2004, leg. A. Ryvkin; 2 우 (CAT, as G. dentatum), Selemdzhinskiy District, left bank of Byssa River about $2 \mathrm{~km}$ up-stream of Kukuya Rill mouth, 310 m a.s.1., 11.VI.2007, leg. E. Veselova \& A. Ryvkin; $2 O^{7} O^{7}, 6$ 우 (ZMMU, as G. dentatum), Khinganskiy N.R., near Antonovskoye, forest, in moss, 1.VIII.1981, leg. unknown; 1 P (CAT, as G. dentatum) Blagoveshchensk, near Verkhneblagoveshchensk, 14.V.-4.VIII.1999, leg. V. Dubatolov; 1 9 (ZMMU, as G. dentatum), Maritime Prov. (= Primorskiy Krai), Khankayskiy District, env. of Turiy Rog, leg. E. Budris; 1 (ZMMU, as G. dentatum), Sikhote-Alin Mts, near Dalnegorsk, 19.VII.1986, leg. B. Zherikhin \& V. Gromov; 1 (ZMMU, as G. dentatum), Shanduy, 12.VII.1978, leg. D. Shcherbakov; $30^{7} \sigma^{7}, 6$ 우 (ZMMU, as G. famelicum), Krasnoyarsk Prov., Nizhnyaya Tunguska River, near Tura, Carex swamp around lake, sweeping, 29.VIII.1982, leg. V. Zherikhin \& K. Eskov.

REMARKS. As noted earlier, the Gnathonarium samples from the Geysers Valley in Kamchatka, as well as from the adjacent continental territories of the Far East and even from central Siberia, all determined as G. dentatum [Eskov, 1992, in part; Eskov \& Marusik, 1992; Marusik et al., 1993; Tanasevitch, 2006, 2008; Tanasevitch \& Trilikauskas, 2004; Trilikauskas \& Tanasevitch, 2006], occupy a position intermediate, as it were, between $G$. dentatum and $G$. suppositum, combining the characters of both species. The shapes of the male carapace and chelicerae from these areas approaches those of G. suppositum (cf. Figs 1-6 \& Figs 7-12, 49-51), but the male palpal tibia and epigynal structure appear to be more similar to G. dentatum (cf. Figs 2227, 65-67 \& Figs 52-64; Figs 68-79 \& 80-94).
This observation implies two possible explanations. Either the Siberian and Far Eastern populations of $G$. dentatum vary geographically so as to show a "suppositum"-like shape of the male carapace or central and eastern Siberia, as well as the continental territories of the Far East are areas of the hybridization of these to closely related species.

It is noteworthy that a "suppositum"-like shape of the male carapace is occasionally found in European populations of $G$. dentatum as well (see Fig. 40), but the available data are still too scarce to warrant a more detailed discussion.

DISTRIBUTION. The species is widely distributed at least in the West Palaearctic, occurring also in the mountains of southern Siberia.

Gnathonarium taczanowskii (O. Pickard-Cambridge, 1873)

Figs 95-104.

1873 Erigone taczanowskii O. Pickard-Cambridge: 443, pl. 41, fig. $10,0^{7}$.

1923 Gongylidium columbianum Emerton: 238, figs 2 a-c, or, MCZ, type examined, syn.n.

TYPE MATERIAL EXAMINED. Gongylidium columbianum Emerton, 1923, 1 ऽ, designated here as lectotype, MCZ \#20849.

COMPARATIVE MATERIAL EXAMINED. Gongylidium taczanowskii: $2 \sigma^{\top} \sigma^{\top}, 3$ ㅇ, 1 ○ subad. (CAT, as G. famelicum), CANADA, Saskatchewan, Besnard Lake, $55^{\circ} 25^{\prime} \mathrm{N} 106^{\circ} 00^{\prime} \mathrm{W}$, rocky beach, under rocks \& litter, 27.V.1970, leg. D. Buckle; $5 \sigma^{7} \sigma^{7}, 6$ 우 (CDB, as G. famelicum), Alberta, Spray Lakes Reservoir, $50^{\circ} 59^{\prime} \mathrm{N}$ $115^{\circ} 21^{\prime} \mathrm{W}$, shingle beach, $1700 \mathrm{~m}$ a.s.1., 19.VII.1990, leg. D. Buckle; $3 \sigma^{\top} \sigma^{\top}, 2$ + 2 (ZMMU), RUSSIA, Chukotka, environs of Lavrentiya, Chigitun River, IX.1991, N. Vekhov; $11 \sigma^{7} \sigma^{7}, 14$ q0 (CAT), Kamchatka Peninsula, Milkovo, Kamchatka River flow, Betula forest, litter, 23-24.VIII.1987, leg. A. Tanasevitch; $4 \sigma^{\top} \sigma^{\top}, 5$ 우 (CAT), Maritime Prov. (= Primorsky Krai), Sikhote-Alin' Mts, Dalnegorsk, 19.VII.1986, leg. V. Zherikhin \& V. Gromov; $3 \sigma^{7} \sigma^{7}$, 1 q (CAT), Amurskaya Area, Norskiy N.R., left confluent of Nora River, upstream of Mt Gryashchinskaya, true mosses and plant debris, 26.VIII.2004, leg. A. Ryvkin; 1 (CAT), Norskiy N.R., shingle-clayish right bank of Nora River under rocky denudation at foot of Mt Ostraya, 23.VIII.2004, leg. A. Ryvkin; $3 \sigma^{\top} \sigma^{\top}, 2$ 우 (CAT), Burunda River bank, $2.5 \mathrm{~km}$ below Burunda Cordon, on soil, in organic debris on wet sand, and in plant debris, 15.IX.2004, leg. A. Ryvkin; $1 \overbrace{}^{7}$ (CAT), same, Burunda River, $1 \mathrm{~km}$ below Burunda Cordon, mosses and leaf litter under Salix sp. near base of shingle-sandy spit, 25.IX.2004, leg. A. Ryvkin; $3 \sigma^{7} \sigma^{7}, 1$ (CAT), rill (left confluent of Nora River) upstream of Mt Gryashchinskaya, true mosses and plant debris, 26.VIII.2004, leg. A. Ryvkin; 10 (CAT), Nora River near Maltsevskiy Cordon, mosses and leaf litter, 2.IX.2004, leg. A. Ryvkin; $1 \sigma^{7}$ (ZMMU), Khinganskiy N.R., near Antonovskoye, forest, in moss, 1.VIII.1981, leg. unknown; $4 \bigcirc^{7} \sigma^{\top}, 8$ 우 (CAT), Khabarovsk Area, Okhotsk, airport, 26.VIII.1985, leg. V. Zherikhin; $2 \sigma^{\top} \sigma^{\top}, 10$ 우 (CAT), Bureinskiy N.R., VII.2005, leg. L. Trilikauskas; ca $60 \bigcirc^{7} \sigma^{7} \&$ + 9 (ZMMU), Krasnoyarsk Prov., Evenk Autonomous Region, bank of Taymura River between Neptene and Chambe rivers mouth, 9-13.VIII.1982, leg. K. Eskov; $2 \sigma^{\top} \sigma^{7}, 1$ + (CAT), Kemerovo Area, near Mezhdurechensk, left bank of Usa River (3 km upstream of its mouth), 23.IX.1997, leg. R. Dudko \& I. Lyubechansky; $12 \sigma^{7} \sigma^{\top}, 14$ 우 (CAT), Tyumen' Area, Polar Urals, Sob' River basin (ca 66 $54^{\circ} \mathrm{N}$, $65^{\circ} 43^{\prime} \mathrm{E}$ ), environs of Rayiz Mts, VI-VII, 1982-1986, leg. A. Tanasevitch; 1 フ , 5 우 (CAT), Altay Republic, Teletskoye Lake, Altayskiy N.R., Lake Chiri, bank of Lake Teletskoye, under stones, $500 \mathrm{~m}$ a.s.1., 27.VII.1997, leg. A. Tanasevitch \& S. Golovatch (new record); ca $20 \sigma^{\top} \sigma^{\top} \&$ \& , (CAT), near Lake Teletskoye, Kebezen', 400-500 m a.s.1., 22.VIII.1998, leg. Veryaskina; 1 o', 1 (CAT), KAZAKHSTAN, East Kazakhstan Area, 25 km NE of Ust'-Ka- 


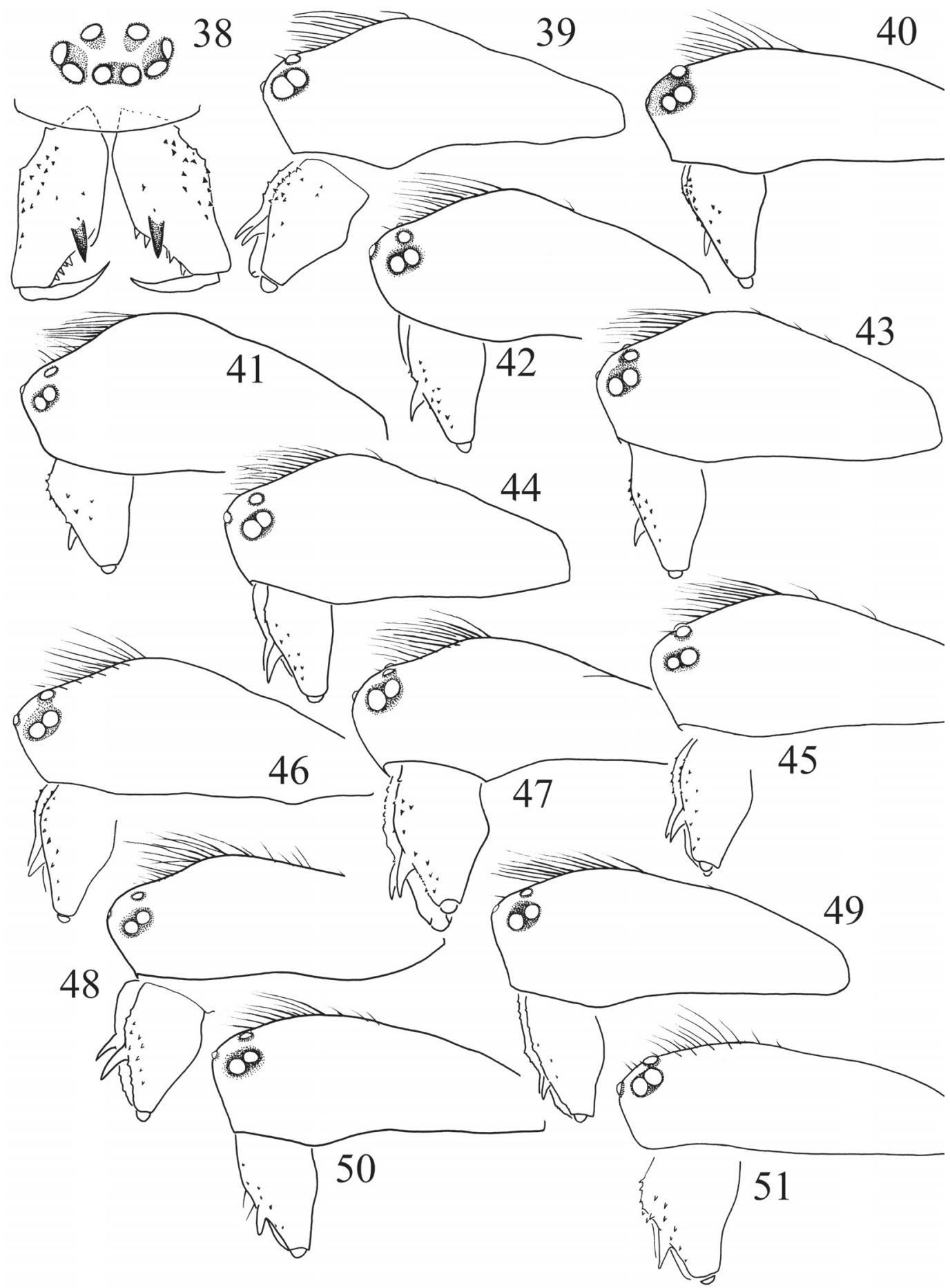

Figs 38-51. Male carapace of Gnathonarium dentatum (Wider, 1834) (38-48) \& G. aff. dentatum (49-51). 38 — frontal view; 3951 — lateral view: 39 - specimen from Lake Kinneret, Israel; 40 - specimen from Hobro, Denmark; 41 - specimen from Poti, Georgia; 42 - specimen from Alexeevka, Azerbaijan; 43 - specimen from Yarodar, Kyrgyzstan; 44 — specimen from Osh City, Kyrgyzstan; 45 - specimen from Karakalpak teppe, Uzbekistan; 46 - specimen from Semenovka, eastern Kazakhstan; 47 — specimen from Yuganskiy N.R., Tyumen' Area; 48 - specimen from Ermakovskoye, Krasnoyarsk Prov.; 49 - specimen from Tura, Evenkia; 50 specimen from Khinganskiy N.R., Amurskaya Area; 51 - specimen from Shanduy, Maritime Prov. Not to scale.

Рис. 38-51. Карапакс самца Gnathonarium dentatum (Wider, 1834) (38-48) \& G. aff. dentatum (49-51). 38 - вид спереди; 39 51 - вид сбоку: 39 - экземпляр с оз. Киннерет, Израиль; 40 - экземпляр из Хобро, Дания; 41 — экземпляр из Поти, Грузия; 42 экземпляр из Алексеевки, Азербайджан; 43 - экземпляр из Яродара, Киргизия; 44 - экземпляр из Ош, Киргизия; 45 экземпляр из Каракалпакской степи, Узбекистан; 46 - экземпляр из Семеновки, Восточный Казахстан; 47 - экземпляр из Юганского заповедника, Тюменская обл.; 48 - экземпляр из Ермаковское, Красноярский край; 49 - экземпляр из Туры, Эвенкия; 50 - экземпляр из Хинганского заповедника, Амурская обл.; 51 - экземпляр из Шандуй, Приморье. Не в масштабе. 


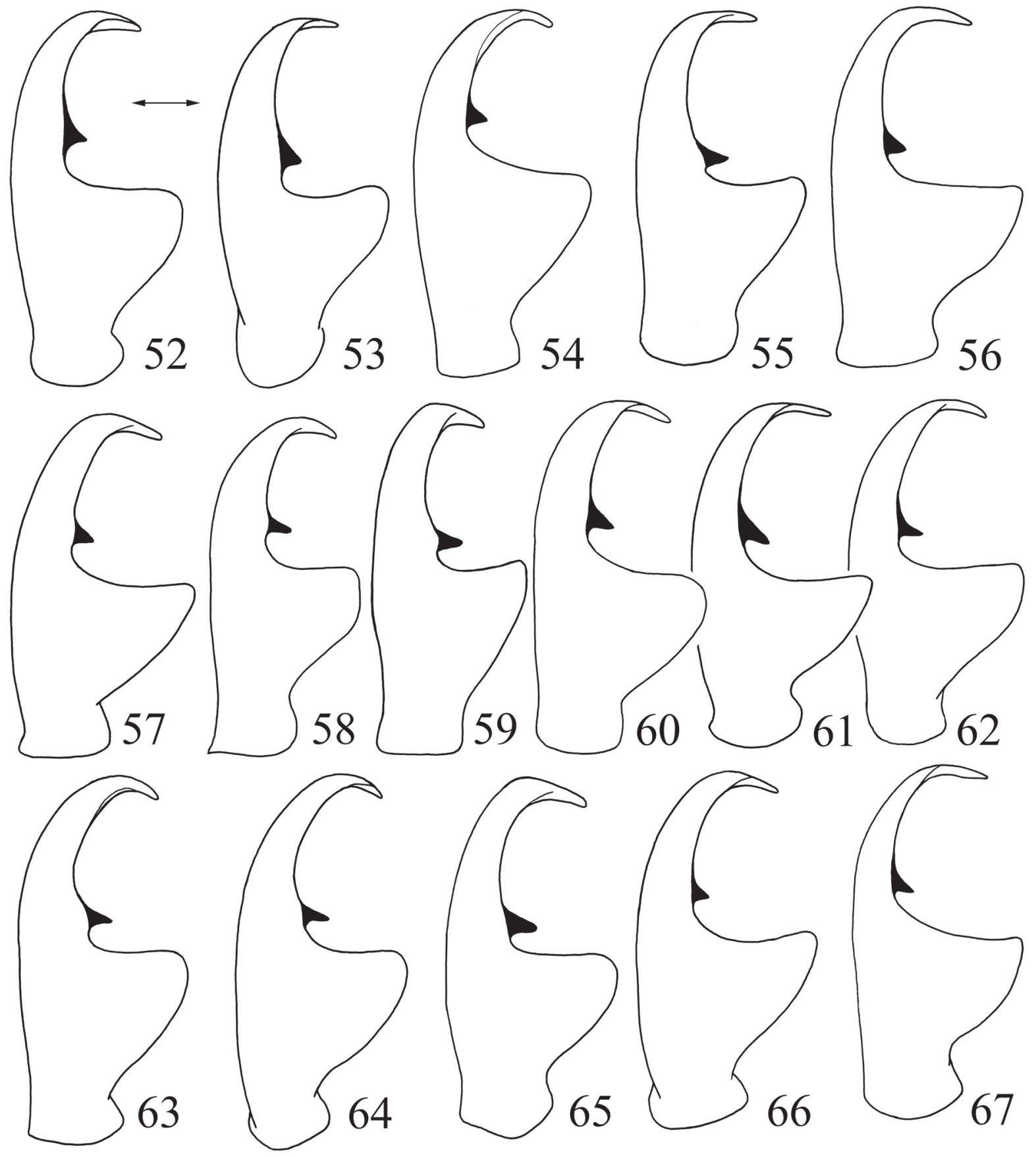

Figs 52-67. Male left palpal tibia of Gnathonarium dentatum (Wider, 1834) (52-64) \& G. aff. dentatum (65-67): 52, 53 - specimen from Hobro, Denmark, different aspects; 54 - specimen from Lake Kinneret, Israel; 55 - specimen from Yuganskiy N.R., Tyumen' Area; 56 - specimen from Semenovka, Kazakhstan; 57 - specimen from Poti, Georgia; 58, 59 - specimen from Alexeevka, Azerbaijan, different aspects; 60 - specimen from Karakalpak steppe, Uzbekistan; 61 - specimen from Osh City, Kyrgyzstan; 62 - specimen from Yarodar, Kyrgyzstan; 63, 64 - specimens from Ermakovskoye, Krasnoyarsk Prov.; 65 — specimen from Tura, Evenkia; 66 — specimen from Shanduy, Maritime Prov.; 67 - specimen from Khinganskiy N.R., Amurskaya Area. Arrows indicate which details belong to the same specimen. Not to scale.

Рис. 52-67. Голень левой пальпы самца Gnathonarium dentatum (Wider, 1834) (52-64) \& G. aff. dentatum (65-67): 52, 53 экземпляр из Хобро, Дания, разные аспекты; 54 - экземпляр с оз. Киннерет, Израиль; 55 - экземпляр из Юганского заповедника, Тюменская обл.; 56 - экземпляр из Семеновки, Казахстан; 57 - экземпляр из Поти, Грузия; 58, 59 - экземпляр из Алексеевки, Азербайджан, разный аспект; 60 - экземпляр из Каракалпакской степи, Узбекистан; 61 - экземпляр из Ош, Киргизия; 62 экземпляр из Яродара, Киргизия; 63, 64 - экземпляры из Ермаковское, Красноярский Край; 65 - экземпляр из Туры, Эвенкия; 66 - экземпляр из Шандуй, Приморье; 67 - экземпляр из Хинганского заповедника, Амурская обл. Стрелки указывают на один и тот же экземпляр. Не в масштабе. 

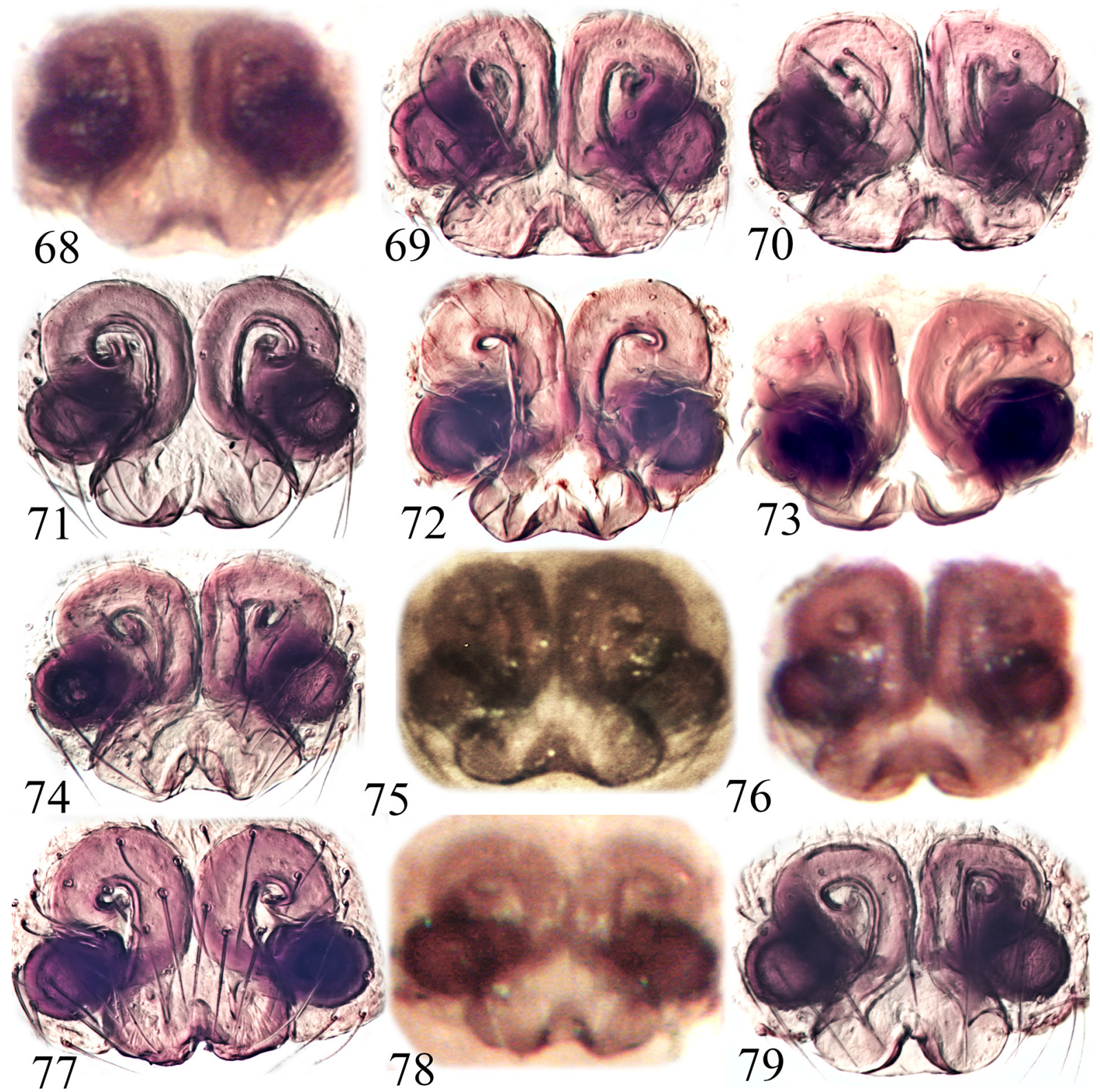

Figs 68-79. Epigyne (ventral view) of Gnathonarium dentatum (Wider, 1834): 68 — specimen from Lake Kinneret, Israel; 69, 70 specimen from Ugory, Kostroma Area, different aspects; 71 — specimen from Yuganskiy N.R., Tyumen’ Area; 72, 73 — specimens from "Missiya”, Tyumen' Area; 74 - specimen from Poti, Georgia; 75 — specimen from Alexeevka, Azerbaijan; 76 - specimen from Santash Valley, Kyrgyzstan; 77 — specimen from Koilyu Valley, Kyrgyzstan; 78 - specimen from Osh City, Kyrgyzstan; 79 — specimen from Karakalpak steppe, Uzbekistan. Not to scale.

Figs 68-79. Эпигина (вид снизу) Gnathonarium dentatum (Wider, 1834): 68 - экземпляр с оз. Киннерет, Израиль; 69, $70-$ экземпляр из Угоры, Костромская обл., разный аспект; 71 - экземпляр из Юганского заповедника, Тюменская обл.; 72, 73 экземпляры «Миссия», Тюменская обл.; 74 - экземпляр из Поти, Грузия; 75 - экземпляр из Алексеевки, Азербайджан; 76 экземпляр из ущелья Санташ, Киргизия; 77 - экземпляр из ущелья Койлю, Киргизия; 78 - экземпляр из Ош, Киргизия; 79 экземпляр из Каракалпакской степи, Узбекистан. Не в масштабе.

menogorsk, Ulba River, Topikha, 300-400 m a.s.1., 11-13.VIII. 1997, leg. R. Dudko \& V. Zinchenko (new record); 2 우 (CAT) ca $35 \mathrm{~km}$ NNE of Leninogorsk, Uba River Valley, $1100 \mathrm{~m}$ a.s.1. taiga, 15 \& 16.VI.1997, leg. R. Dudko; 3 o $^{7} 0^{2}, 2$ 우 (CAT), Samarskoye District, Kaindinskiy Bor, $700-900$ m a.s.1., 2628.VIII.1985, leg. A. Fedorov (new record); $1 \sigma^{7}, 2$ 우 CAT), Bukhtarma River, mouth of Sarymsakty River, $600 \mathrm{~m}$ a.s.l., 1.VIII.1996, leg. R. Dudko \& V. Zinchenko (new record); $10^{7}, 2$ 우 (CAT), Kalbinskiy Mt. Ridge, upper reaches of Kopirli River,
$20 \mathrm{~km}$ SSE of Verkhnyaya Tainta, $1200 \mathrm{~m}$ a.s.1., 10.V.1999, leg. R. Dudko, V. Zinchenko, I. Lyubechansky; 1 + (CAT), Pavlodar Area, Mayskoye District, Lake Koktas, V.1990, leg. O. Lyakhov (new record).

REMARKS. G. taczanowskii was originally described from Lake Baikal, Siberia [O. Pickard-Cambridge, 1873], in Erigone, and has since been listed under various names [see Platnick, 2013]. 
An examination of the type of $G$. columbianum Emerton, 1923, described from Terrace, British Columbia, shows that this species is conspecific to $G$. taczanowskii and should be considered as its new subjective junior synonym (syn.n.). The synonymy proposed by Eskov [1988: 105], i.e., G. columbianum = $G$. suppositum, is wrong.

DISTRIBUTION. The Palaearctic: from the Urals through entire Siberia to Chukotka in the east, Mongolia, Tibet \& China in the south; in the Nearctic the easternmost locality is in Manitoba [Benell-Aitchison \& Dondale, 1990].

PATTERN. East Palaearctic - Nearctic.

\section{Pelecopsis laptevi Tanasevitch et Fet, 1986}

1986 Pelecopsis laptevi Tanasevitch \& Fet: 35, fig. 2: 1-4, ○, ZIN, examined.

1989 P. laptevi. - Tanasevitch: 145, figs 174-177, ○’ \& ㅇ, examined.

2008 P. steppensis Gnelitsa: 137, figs 1 (a-e), 2 (a-b), 3 (a-d), $\checkmark$ \& $\sigma^{\circ}$, syn.n.

2008 P. laptevi. — Gnelitsa: 139, figs 1 (f-j), 2 (c-d), $3(\mathrm{e}-\mathrm{h})$, $\sigma^{7} \&$ ?

2013 P. laptevi. - Polchaninova: 91, reference.

TYPE MATERIAL EXAMINED. The types of Pelecopsis steppensis Gnelitsa, 2008 are not in ZMMU, although claimed to have been deposited there. P. laptevi: holotype $\sigma^{7}$ (ZIN), TURKMENISTAN, Plateau Badkhyz, Kepele Cordon, under Pistacia, 18.I.1978, leg. Kh. Atamuradov; paratypes: $1 \sigma^{7}(\mathrm{ZIN})$, same, Kyzyldjar Cordon, in litter under Ephedra, 27.I.1978, leg. V. Krivokhatsky; 1 ठ (ZIN), Kopetdagh Mts, Aidere Valley, in litter under Acer, 20 30.III.1980, leg. V. Fet.

COMPARATIVE MATERIAL EXAMINED. $20 \sigma^{\gamma} \sigma^{7}, 10$ 우 (CAT), RUSSIA, Rostov Area, northern coast of Taganrog Bay, Azov Sea, Neklinovsky District, Beglitskaya Spit, sandy beach, 30.III-20.IV.2012, leg. A. Ponomarev; 1 o (CAP), same, steppe vegetation on beach, 1.VII.2010, leg. A. Ponomarev; $1 \sigma^{\prime}$ (CAP), Azov District, southern coast of Taganrog Bay, Azov Sea, near Stafanidinodar, sandy beach, 17.VI.2010, leg. A. Ponomarev; 3 ofo (CAT), KAZAKHSTAN, Mangistau (= Mangyshlak) Area, Karakiya (= Eraliev) District, Ustyurt Plateau, ca $47 \mathrm{~km}$ E of Akkuduk Ruins, Ustyurt N.R., environs of Kendirli Well, ca $42^{\circ} 57^{\prime} 17.4^{\prime \prime} \mathrm{N}$ $54^{\circ} 41^{\prime} 00.0^{\prime \prime} \mathrm{E}$, ca $110 \mathrm{~m}$ a.s.1., 21.V.1989, leg. A. Zyuzin; $4 \sigma^{\top} \sigma^{\top}, 4$ Oᄋ (ZMMU), 3 O $^{\top}, 3$ OO (SMF 34796), TURKMENISTAN, Mary Area, Serhetabad [= Kushka] District, Badkhyz Plateau, Badkhyz N.R., environs of Kyzyldjar Cordon, ca $35^{\circ} 49^{\prime} 31.0^{\prime \prime} \mathrm{N}, 61^{\circ}$ $50^{\prime} 56.0^{\prime \prime} \mathrm{E}$, ca $720 \mathrm{~m}$ a.s.1., 17-28.II.1978, leg. V. Krivokhatsky; 1 + (MHNG), IRAN, Khorasan, Emamgholi ( $\left.37^{\circ} 26^{\prime} \mathrm{N}, 58^{\circ} 30^{\prime} \mathrm{E}\right)$, 15.VII.1974, leg. A. Senglet.

REMARKS. This species was originally described from males from Plateau Badkhyz and SW-Kopetdagh Mts, Turkmenistan [Tanasevitch \& Fet, 1986]; later the female was described from Turkmenistan as well [Tanasevitch, 1989]. P. steppensis was based on two males and two females from the Ukraine, being treated by its author as closely related to $P$. laptevi [Gnelitsa, 2008]. The abundant material studied here shows the absence of any reliable differences that would distinguish $P$. steppensis as a valid species. Small deviations are only observed in the shape of the male carapace and in some parts of the genitalia. Thus, among the European and Turkmenistan populations males can be found showing the sulci either rounded or slightly elongated; with the posterodorsal outgrowth on the cymbium being either straight or slightly inclined. The minute differences in the membranous distal suprategular apophysis and in the shape of the small tubercle in the proximal part of the radix, etc., they all lie well within the range of individual or populational variation. Such variations are best traceable using abundant material, versus the very small type series $\left(2 O^{7} O^{7} \& 2\right.$ \& 9 ) studied by Gnelitsa [2008]. In addition, some of the differences might be imaginary, depending on aspect.

DISTRIBUTION. Southern part of the Russian Plain: Ukrainian steppes and Crimea; Rostov Area in Russia; Ustyurt Plateau in Kazakhstan, Badkhyz Plateau and Kopetdagh Mts in Turkmenistan, as well as Khorasan in Iran.

PATTERN. Sarmato-Turanian.

\section{Wabasso replicatus (Holm, 1950)}

1986 Diplocentria replicata. - Eskov: 185, examined.

1988 W. quaestio. - Eskov: 136, reference.

1992 W. quaestio. - Eskov: 80, misidentification, examined.

$1998 W$. quaestio. - Koponen et al.: 116, misidentification, examined

1994 W. quaestio. — Eskov \& Marusik: 89, misidentification, examined.

2005 W. quaestio. - Tanasevitch: 78, misidentification, examined.

2007 W. replicatus. - Tanasevitch \& Koponen: 322, examined.

2010 W. replicatus. - Tanasevitch \& Rybalov: 49, examined. MATERIAL EXAMINED. $1 \sigma^{\top}$ (CAT), RUSSIA, Murmansk Area, Kola Peninsula, Teriberka, Carex-Sphagnum-Eriophorum bog with Salix sp. stands, Comarum palustre, 6.VII.2008, leg. L. Rybalov \& V. Semenov; 2 우 (CAT), same, Carex-Sphagnum-Eriophorum bog with Salix sp. stands, 4-6.VIII.2009, leg. V. Piryugin; 5 우 (CAT), Arkhangelsk Area, Nenets Autonomous Region, Bolshezemelskaya Tundra, Shapkina River (ca $\left.67^{\circ} 49^{\prime \prime} \mathrm{N}, 56^{\circ} 08^{\prime \prime} \mathrm{E}\right)$, bog on lake bank, in Sphagnum, 15.VII.1984, leg. A. Tanasevitch; 4 O $^{7}, 7$ 우 (CAT), Tyumen' Area, environs of Noyabrsky, Salix bushes along brook, litter, 15.VIII.2000, leg. A. Tanasevitch; $1 \sigma^{7}, 1$ \% (ZMMU), Yamalo-Nenets Autonomous Region, Yamal Peninsula, environs of Shchyuchye, swamp, in Polytrichum, VIII.1980, leg. A. Tikhomirova \& E. Veselova; $2 \sigma^{7} \sigma^{7}, 3$ 우 (CAT), Krasnoyarsk Prov., Tsentralno-Sibirskiy N.R., Yuzhnyi Cordon, 2.X.1994, leg. A. Ushakov; $1 \sigma^{\top}$ (CAT), Tsentralno-Sibirskiy N.R., Birobchina River, 3.VII.1988, leg. A. Ryvkin; $1 \sigma^{\top}$ (ZMMU), Evenk Autonomous Region, Birobchana River flow, 5.VII.1988, leg. A. Ryvkin; 1 ○ (ZMMU), Tuva Republic, Azas N.R., 1.VI.1990, leg. A. Ryvkin.

REMARKS. After Merrett \& Dawson [2005] revalidated Wabasso replicatus (Holm, 1950), we have checked all available material of $W$. quaestio (Chamberlin, 1949) from the Euro-Siberian region deposited in ZMMU and CAT. As a result, all of them actually refer to $W$. replicatus. Therefore, $W$. quaestio seems to be restricted to the eastern Nearctic, without any reliable records in the Palaearctic.

DISTRIBUTION. Europe: Great Britain, Iceland, Fennoscandia, European tundra; Siberia: southern Yamal Peninsula, west, central and south (Tuva \& Sayan) Siberia.

PATTERN. Euro-Siberian.

Agyneta birulaioides Wunderlich, 1995

TYPE MATERIAL EXAMINED. Museum labels: Meioneta birulaioides, $1 \sigma^{7}$ paratype, SMF 60191, MONGOLIA, Dundgovi, Mittelgobi Aimak, Choot bulag, between Somon Chuld and Somon 

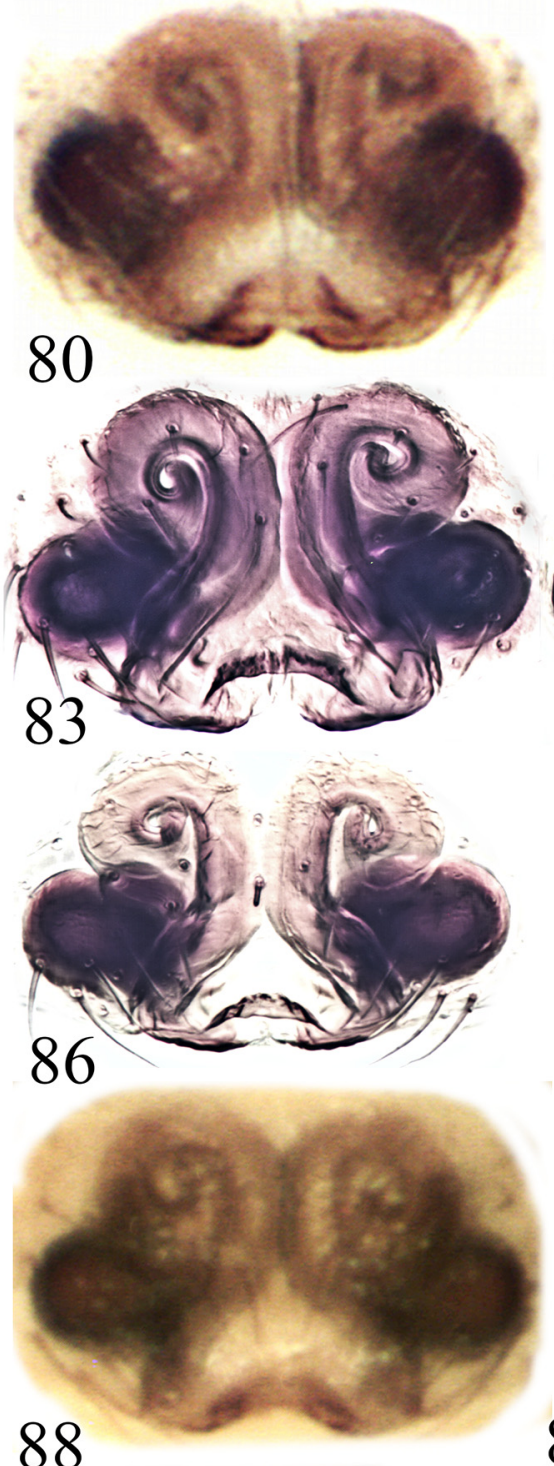

88

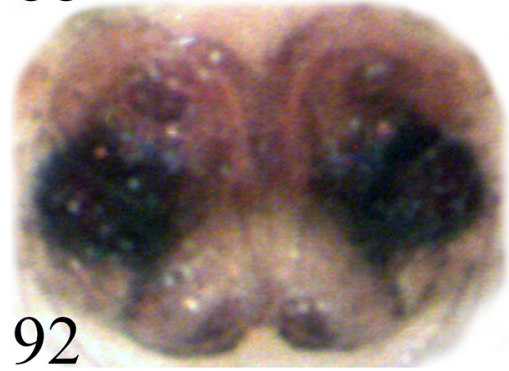

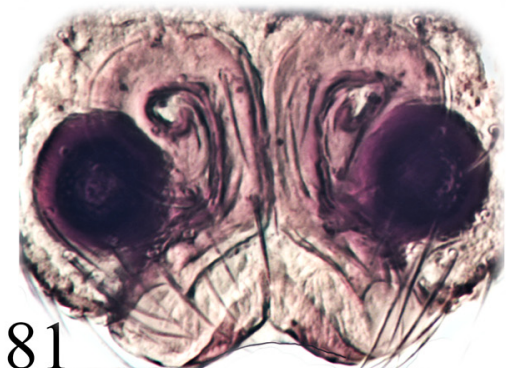

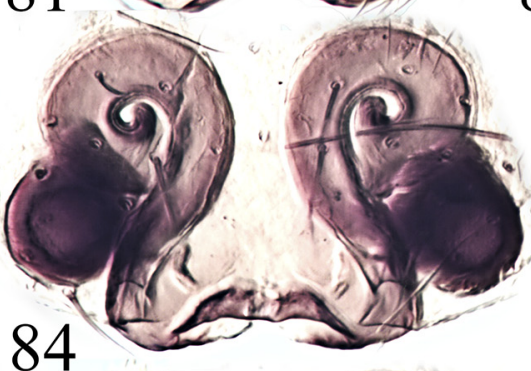

82
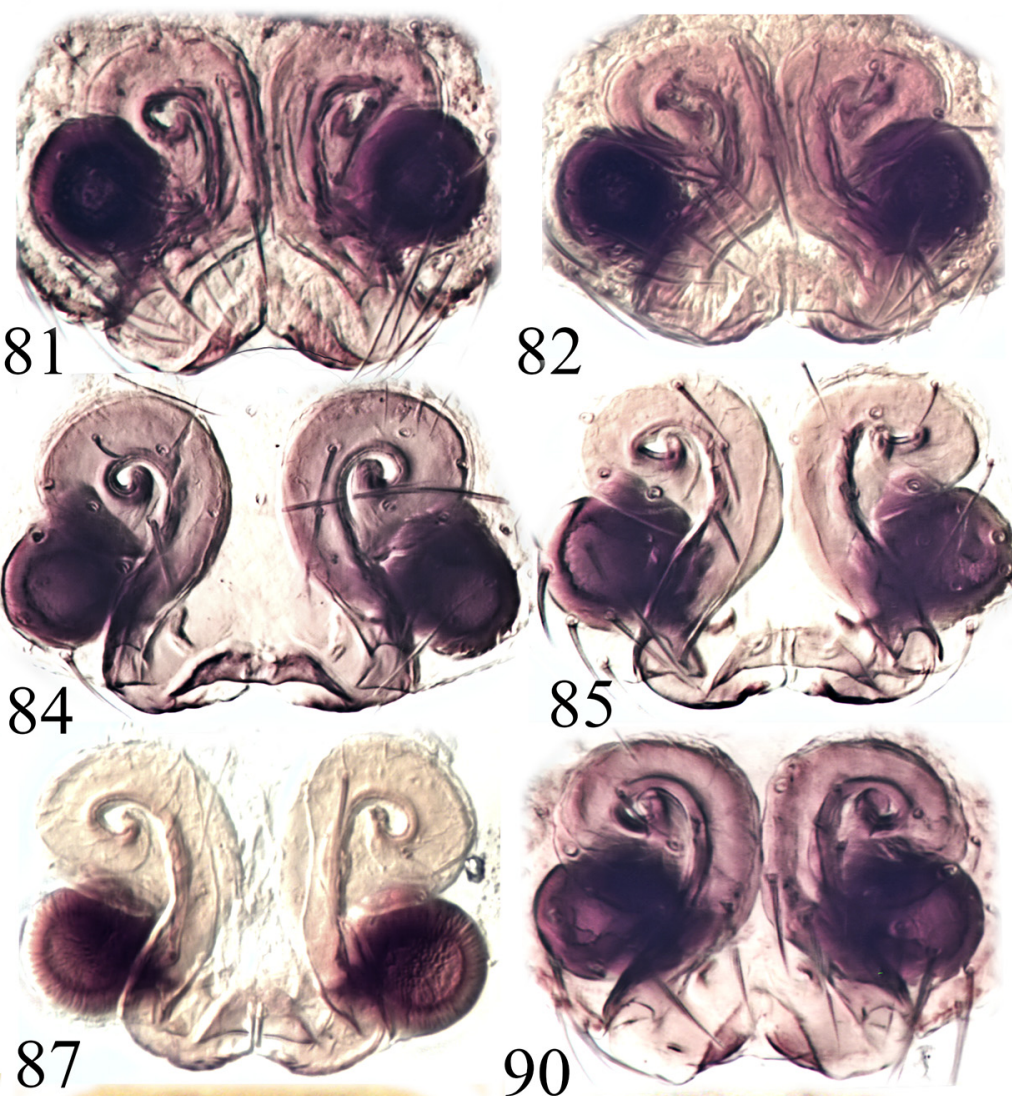

85
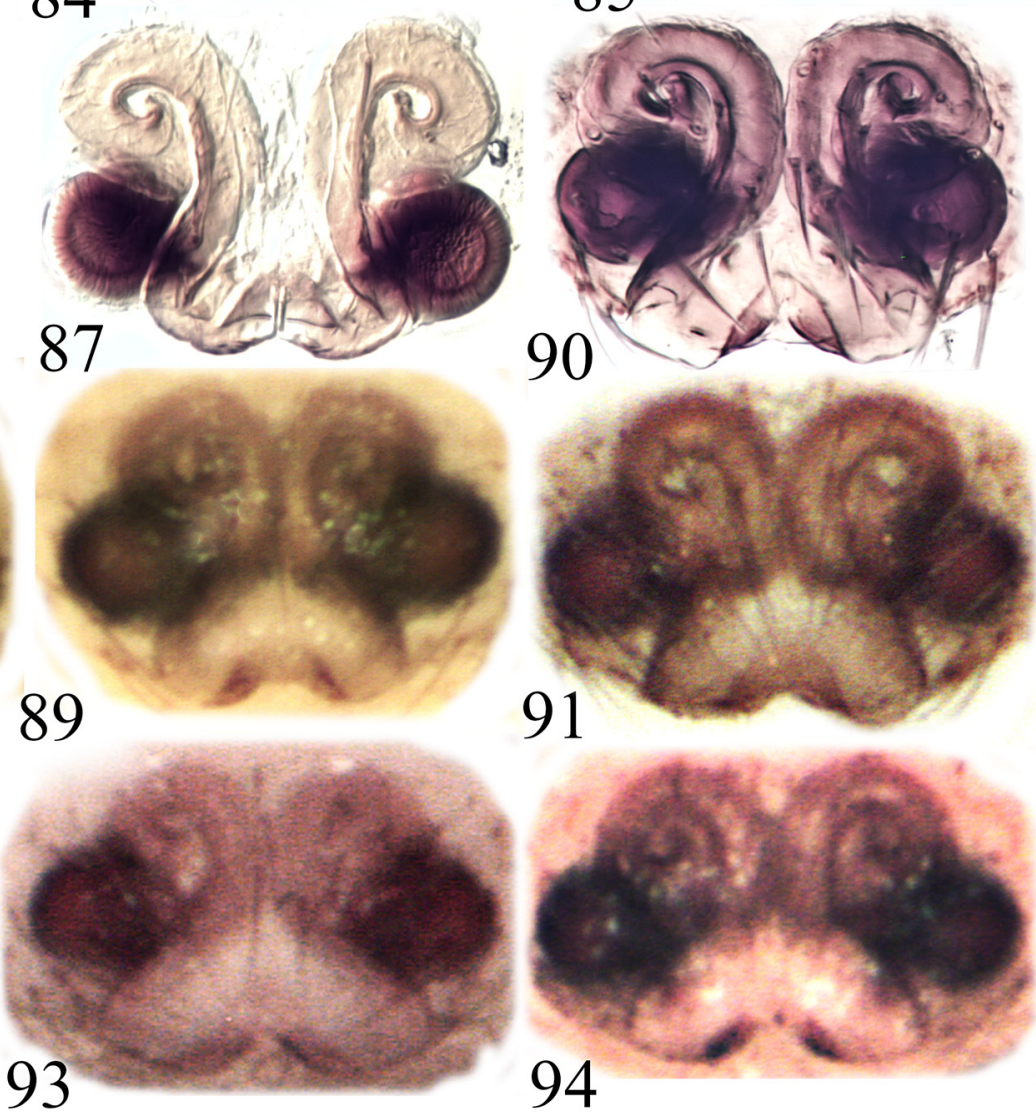

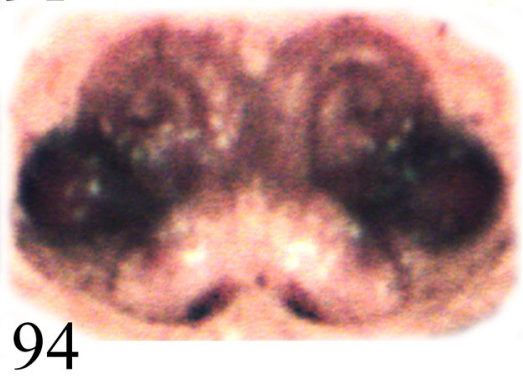

Figs 80-94. Epigyne (ventral view) of Gnathonarium aff. dentatum (Wider, 1834): 80 — specimen from Tura, Evenkia; 81, 82 — specimens from Turiy Rog, Maritime Prov.; 83-89 — specimens from Geysers Valley, Kamchatka; 90 - specimen from Blagoveshchensk, Amurskaya Area; 91- specimen from Khinganskiy N.R., Amurskaya Area; 92 - specimen from Byssa River, Amurskaya Area; 93, 94 - specimens from Chegdomyn, Khabarovsk Prov. Not to scale.

Рис. 80-94. Эпигина (вид снизу) Gnathonarium aff. dentatum (Wider, 1834): 80 - экземпляр из Туры, Эвенкия; 81, 82 — экземпляры из Турьего Рога, Приморье; 83-89 - экземпляры из Долины гейзеров, Камчатка; 90 - экземпляр из Благовещенска, Амурская обл.; 91- экземпляр из Хинганского заповедника, Амурская обл.; 92 - экземпляр с реки Бысса, Амурская обл.; 93 , 94 экземпляры из Чегдомына, Хабаровский Край. Не в масштабе. 

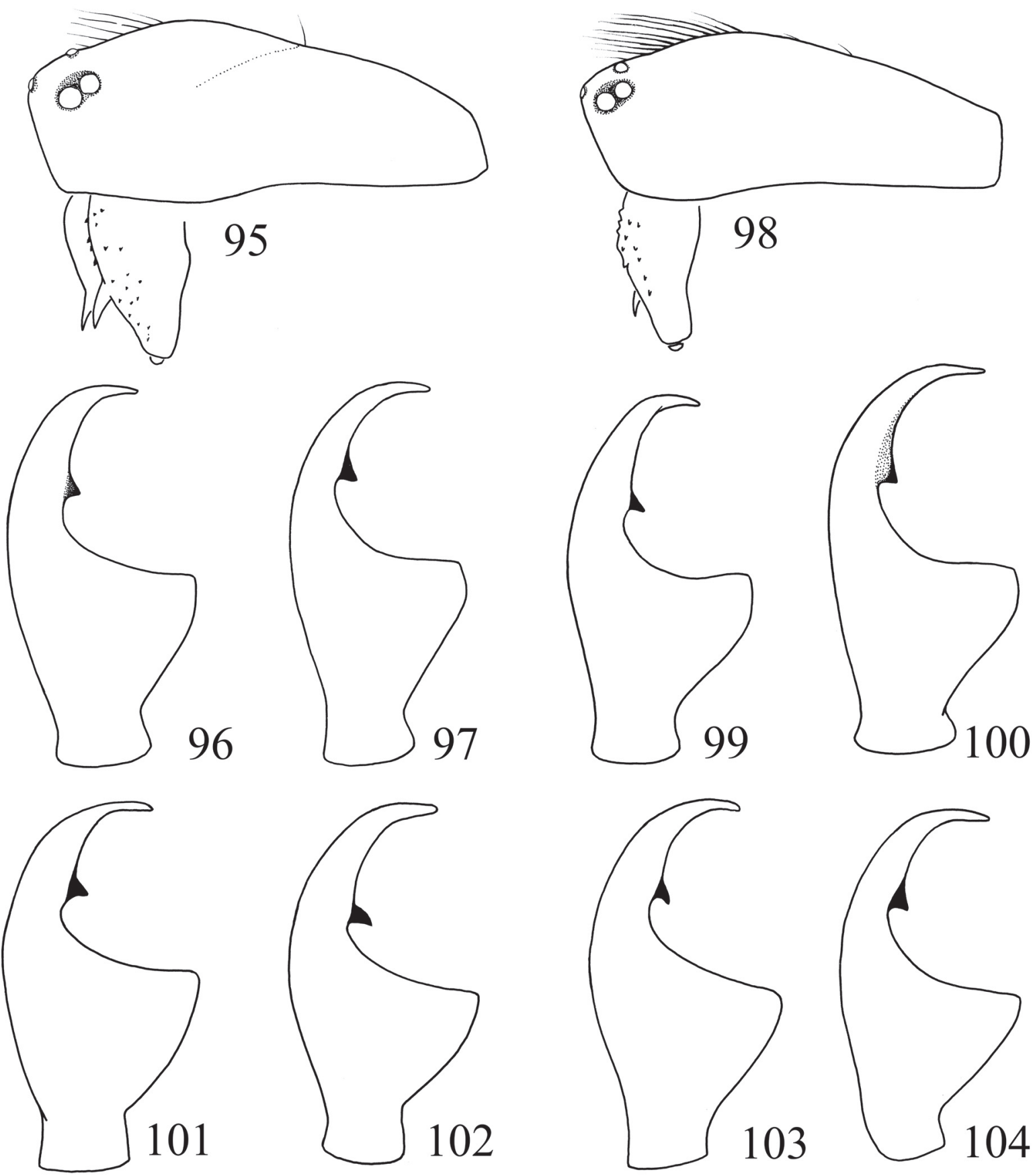

Figs 95-104. Male carapace and male left palpal tibia of Gnathonarium columbianum (Emerton, 1923), type (95-97) \& G. taczanowskii (O. Pickard-Cambridge, 1873) (98-104): 95, 98 - carapace, lateral view; 96, 97, 99-104 - palpal tibia, dorsal view; 95-97 - type from Terrace, British Columbia; 98, 102 - specimen from Norskiy N.R., Amurskaya Area; 99, 100 - specimens from Lake Besnard, Saskatchewan; 101 - specimen from Lavrentiya, Chukotka; 103 - specimen from Dalnegorsk, Maritime Prov.; 104 specimen from Taymura River, Evenkia. Arrows indicate which details belong to the same specimen. Not to scale.

Рис. 95-104. Карапакс и голень левой пальпы самца Gnathonarium columbianum (Emerton, 1923), тип (95-97) \& G. taczanowskii (O. Pickard-Cambridge, 1873) (98-104): 95, 98 — карапакс, вид сбоку; 96, 97, 99-104 — голень пальпы, вид сверху; 95-97 — тип из Террас, Британская Колумбия; 98, 102 - экземпляр из Норского заповедника, Амурская обл.; 99, 100 - экземпляр с оз. Беснард, Саскачеван; 101 - экземпляр из Лаврентия, Чукотка; 103 - экземпляр из Дальнегорска, Приморье; 104 - экземпляр с реки Таймура, Эвенкия. Стрелки указывают на один и тот же экземпляр. Не в масштабе. 
Delgerchangaj, $38 \mathrm{~km}$ ENE of Delgerchangaj, $1480 \mathrm{~m}$ a.s.1., 10.VI.-12.VII.1967, leg. Z. Kaszab, det. J. Wunderlich; $5 \mathrm{O}^{\top} \mathrm{O}^{\top}, 3$ paratypes, SMF 60193, MONGOLIA, Tov, Central Aimak, Bogdo ul, Bugijn az achuj, 1650 m a.s.l., leg. Z. Kaszab, 10.VI.1968, det. J. Wunderlich.

MATERIAL EXAMINED. $1 \overbrace{}^{7}, 1$ (ZMMU), UKRAINE, Kherson Area, Golaya Pristan District, Chernomorskiy N.R., IvanoRybalchanskiy area, Rybachie, salina, 11.V.1999, leg. Prokopenko; 1 \& (ZMMU), same, Solyonoozerskiy area, environs of Geroyskoye, sandy steppe with low herbs, 14.IV.1985, leg. N Polchaninova; $1 \sigma^{\prime \prime}, 1$ ( $(\mathrm{CAT})$, KAZAKHSTAN, South Kazakhstan Area, Almaty Area, Karatal District, $35 \mathrm{~km} \mathrm{NW}$ of $3^{\text {rd }}$ Lake Ushkol (= Lake Shorayakkol), ca $46^{\circ} 08^{\prime} 03.2^{\prime \prime} \mathrm{N} 77^{\circ} 40^{\prime} 45.0^{\prime \prime} \mathrm{E}$, sandy desert ("Zhamanzhal Sands"), from rodent burrows, 30.VII.1988, leg. A. Zyuzin, M. Zarko \& Ch. Tarabaev (all new localities) Earlier identifications of Agyneta birulaioides: $\mathrm{O}^{7} \mathrm{O}^{7} \&$ 우 (ZMMU) RUSSIA, Tuva Republic, 3-5 km N of Kyzyl, 650-900 m a.s.1., $51^{\circ} 46^{\prime} \mathrm{N} 94^{\circ} 27^{\prime} \mathrm{E}, 20.05-21 . I X .1989$, leg. D. Logunov; NE bank of Lake Ubsunur, $50^{\circ} 40^{\prime} \mathrm{N} 92^{\circ} 58^{\prime} \mathrm{E}, 760 \mathrm{~m}$ a.s.1., 12.VI.1989, D Logunov; Sangelen Mt. Ridge, upper reaches of Dzhen-Aryk, $50^{\circ}$ $28^{\prime} \mathrm{N} 95^{\circ} 24^{\prime} \mathrm{E}, 1750-2030 \mathrm{~m}$ a.s.1., 16-18.07.1996, leg. Yu. Marusik

REMARKS. A. birulaioides Wunderlich, 1995 was described from both sexes from the mountainous regions of Middle Gobi, Mongolia [Wunderlich, 1995], later found in Tuva as well [Logunov et al., 1998]. A comparison of the samples of $A$. birulaioides deriving both from the Kherson steppes, Ukraine and a cisBalkhash sand desert with the types of $A$. birulaioides from the Gobi of Mongolia shows their conspecificity. $A$. birulaioides is a nice example of the so called Scythian distribution pattern [Kryzhanovsky, 2002], i.e. the Eurasian steppe belt ranging from the Ukraine in the west to Lake Balkhash, Tuva and Mongolia's Gobi in the east. The species is being reported here as new to the Ukrainian fauna.

\section{Porrhomma boreale (Banks, 1899)}

Figs 105-116.

1899 Gongylidium borealis Banks: 347, fig. 1, ○ \&

1970 Porrhomma nunamo Holm: 194, figs 12-15, or \& 우, NHRS, type examined.

1974 P. kulczynskii Starega: 22, figs 7, 8, photo 3, o, syn.n.

TYPE MATERIAL. The type of Porrhomma kulczynskii Staręga, 1974 has not been re-examined, being absent from the MIZ collection in Warsaw, Poland. Porrhomma nunamo Holm, 1970 NHRS-GULI 000000244, $1 \sigma^{7}$ paratype, Siberia, St. Lawrence Bay, Nunamo, 27/7 1879, Vega-Exp., coll. \#1045, det. Å. Holm.

COMPARATIVE MATERIAL EXAMINED. Earlier identifications of Porrhomma kulczynskii: 1 \%, 1 क (CAT), RUSSIA, Maritime Prov. (= Primorskiy Krai), Ussuriysk District, Ussuriyskiy N.R., Quercus \& Pinus sibirica forest, 12.VII.1977, leg. G. Kurcheva \& E. Mikhaljova; 1 (CAT), Amurskaya Area, Selemdzhinsk District, Byssa River basin NW of "Tyoplyi Klyuch" spa, Salix \& Alnus forest, in moss and litter, 21.VI.2007, leg. E. Veselova \& A Ryvkin (new locality); $4 \bigcirc^{\top} \sigma^{7}$ (CAT), Khabarovsk Prov., Bureinskiy N.R., 22.VII.2005, leg. L. Trilikauskas (new locality). Porrhomma boreale: 1 \%, 3 90 (CAT), Komi Republic, Vorkuta District, environs of Khalmer-Yu, brook channel, under stones, 13-16.VIII.1984, leg. A. Tanasevitch; 1 q (CAT), Tyumen’ Area, Polar Urals, $10 \mathrm{~km}$ NNW of Kharp, bank of Sob' River, under stones, 3.VIII.1981, leg. A. Tanasevitch (new locality); 19 (ZMMU, as $P$. convexum), Krasnoyarsk Prov., middle flow Yenisei River, near Mirnoye, willow shrub formation on river bank, litter, 18.VI.1978, leg. K. Eskov; $1 \mathrm{O}^{7}, 2$ + Krai, Charysh Distr., confluence of Inya \& Belogolosov Korgon rivers, $50^{\circ} 57-59^{\prime} \mathrm{N} 83^{\circ} 39-40^{\prime} \mathrm{E}, 2000-2250 \mathrm{~m}$ a.s.1., 27-28.VI.2011, leg. R. Dudko (new locality).
REMARKS. P. kulczynskii was originally described by two females from Zaisan, Ulaanbaatar, Mongolia. Starega [1974], among others, compared this species to $P$. nunamo Holm, 1970 [ $=$ P. boreale $]$. Amongst the differences from $P$. nunamo, he noted: “... large, distinctly colored eyes, the absence of the long hairs on cephalothorax, as well as the absence of a dorsal spine on femora I". The size of the eyes and the ocular pigmentation vary indeed, albeit insignificantly, whereas the head and face are pubescent only in the male. Examination of the type and comparative material of $P$. boreale and its comparison with the figures and original description of $P$. kulczynskii shows that both these species are conspecific. Therefore, the latter taxon is to be regarded as a new subjective junior synonym of $P$. boreale, syn.n. The absence of a dorsal spine on FeI in $P$. kulczynskii is most likely secondary, as a dorsal spine or spines are present, if not broken-off, in most of the species of Porrhomma Simon, 1884.

In contrast to the numerous Porrhomma species barely distinguishable by the shape of the epigyne, the female of $P$. boreale differs well from the other congeners in the peculiar structure of the vulva, i.e. elongated spiral receptacles which bases give rise to thin secondary vermiform receptacula, the latter embracing like snakes the middle parts of the former. The shape and pubescence of the head of the male carapace are very similar to those in P. magnum Tanasevitch, 2012, this latter species being distributed in the mountains of southern Siberia [Tanasevitch, 2012].

DISTRIBUTION. Polar Urals, western, central, southern and eastern Siberia, northern Mongolia, and Russian Far East in the Palaearctic; Alaska in the Nearctic.

PATTERN. Siberian-Alaskan.

Sibirocyba incerta (Kulczyński, 1916)

1955 Tapinocyba incerta. - Tullgren: 374, figs $75 \mathrm{a}-\mathrm{b}$, + , misidentification.

REMARKS. Tullgren [1955] provided drawings of the epigyne of Tapinocyba incerta Kulczyński, 1916, a species claimed to be new to the Swedish fauna. In fact those illustrations depict the West Palaearctic Tapinocyboides pygmaeus (Menge, 1869). Hence, the range of $T$. incerta, now Sibirocyba incerta, is to be considered as Siberian, ranging from the Polar Urals throughout Siberia to Chukotka in the east, and to southern Siberia (Tuva) in the south.

Tibioploides stigmosus (Xia, Zhang, Gao, Fei et Kim, 2001)

MATERIAL EXAMINED. 2 ㅇ (ZMMU, as Tibioploides sp.n.), Iturup Isl., Dobroye Nachalo Gulf, mixed forest, 13-14.VIII.1994, leg. K. Eskov; 1 ऽ , 5 우 (ZMMU, as Tibioploides sp.), southern Kurile Islands, Kunashir Island, Lake Oliger, mixed forest, 31.VIII.1994, leg. K. Eskov, KU-4; 1 \& (ZMMU), near Yuzhno-Kurilsk, Picea forest, bog, 1.VIII.1994, leg. K. Eskov, KU-9; 2 +क (ZMMU, as Tibioploides sp.n.), Shikotan Isl., Svobodnaya River, mountain forest of Abies \& Betula forest, 8.VIII.1994, leg. K. Eskov, SH-34. 

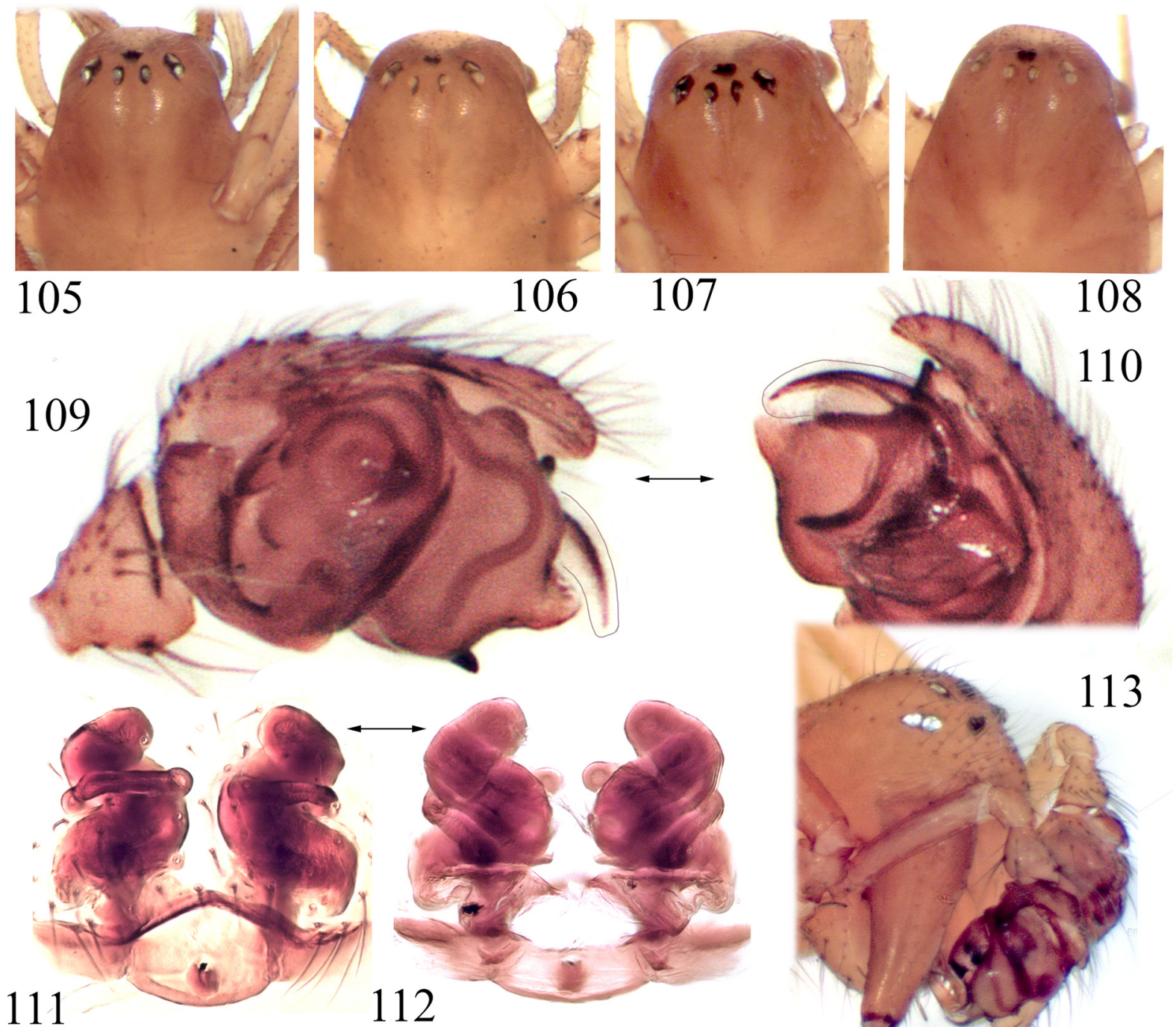

106

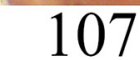

111

112
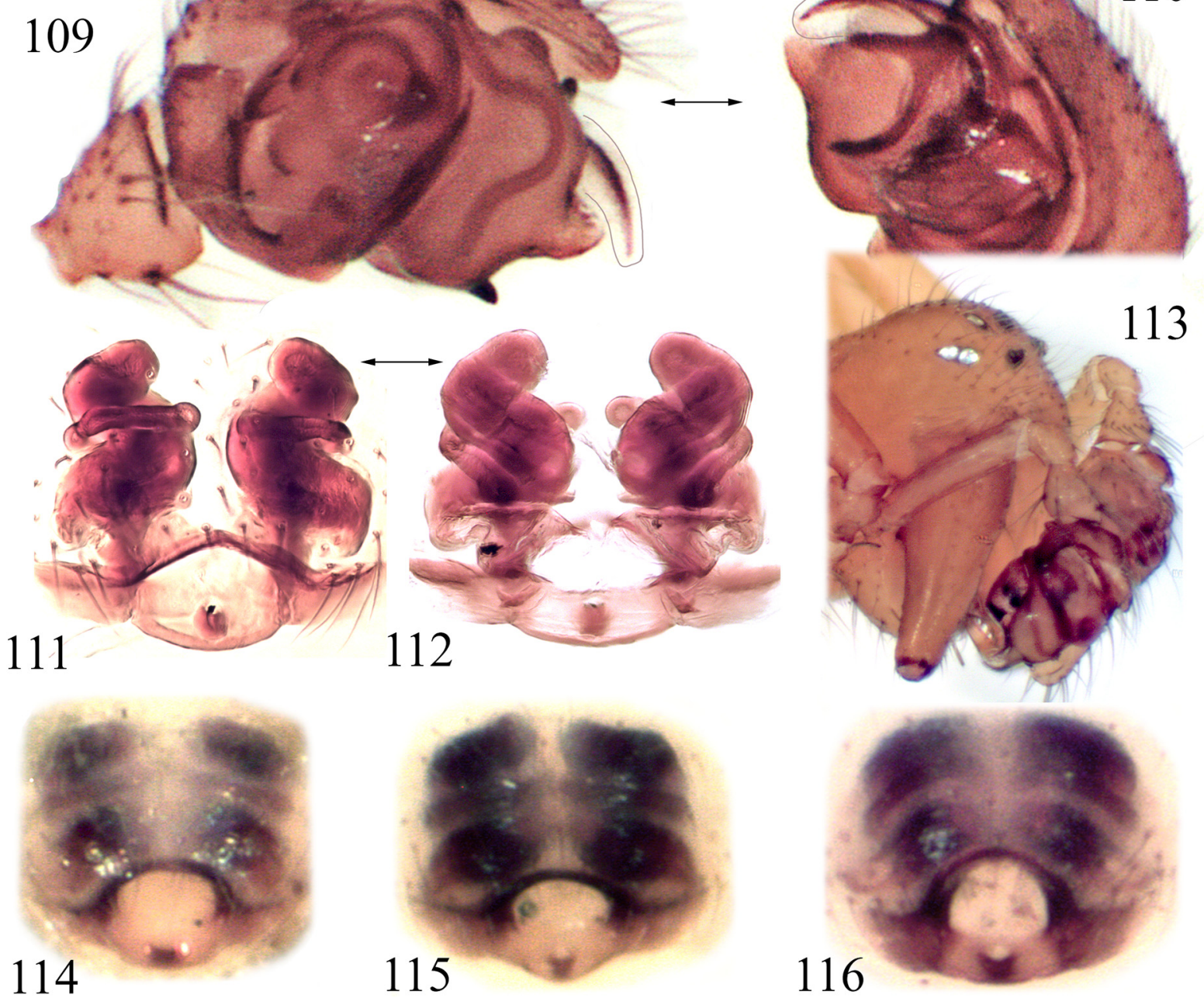

Figs 105-116. Porrhomma boreale (Banks, 1899): 105-108 — head part of female carapace, dorsal view; 109, 110 — male right palp, retrolateral \& prolateral views, relatively; 111, 112 — vulva, ventral \& dorsal views, relatively; 113 — head part of male carapace, lateral view; 114-116 - epigyne, ventral view; 105, 106, 111, 112 — specimens from Bureinskiy N.R., Khabarovsk Prov.; 107, 108, 115 specimens from Ussuriyskiy N.R., Maritime Prov.; 109, 110, 114 — specimens from Polar Urals, Tyumen’ Area; 113, 116 — specimens from Inya River, Altai Mts. Arrows indicate which details belong to the same specimen. Not to scale.

Рис. 105-116. Детали строения Porrhomma boreale (Banks, 1899): 105-108 - головная часть карапакса самки, вид сверху; 109, 110 - правая пальпа самца, ретролатерально и пролатерально, соответственно; 111, 112 - эндогина, вентрально и дорзально, соответственно; 113 - головная часть карапакса самца, вид сбоку; 114-116 - эпигина, вид снизу; 105, 106, 111, 112 экземпляры из Буреинского заповедника, Хабаровский край; 107, 108, 115 - экземпляры из Уссурийского заповедника, Приморье; 109, 110, 114 - экземпляры с Полярного Урала, Тюменская обл.; 113, 116 - экземпляры с реки Иня, Алтайские горы. Стрелки указывают на один и тот же экземпляр. Не в масштабе. 
COMPARATIVE MATERIAL EXAMINED. $10^{7}, 2$ Oᄋ (SMF), China, Qinghai Prov., Bei Shan National Park, 120 km NE Xining, 2300-2700 m a.s.1., 23.V.-8.VI.1996, leg. J. Martens.

REMARKS. This species has hitherto been known from Qinghai and Gansu provinces, China [Tanasevitch, 2006], being new to the Russian fauna.

\section{Mecynargus minutipalpis Gnelitsa, 2011}

1984 Mecynargus longus. - Minoransky \& Ponomarev: 84, misidentification, examined.

1984 M. longus. — Ponomarev \& Minoransky: 234, misidentification, reference.

1988 M. longus. - Ponomarev: 55, misidentification, reference 2011 M. minutipalpis Gnelitsa: 65, $\sigma^{7} \&$ ㅇ․

MATERIAL EXAMINED. 3 우 (CAP, \#40.18.1/2), RUSSIA, Kalmyk Republic, Chernozemelskiy Distr., 40 km S of Komsomolskiy, near Rybachiy, bank of salt lake,16.VI.1974; 1 (CAP, \#40.18.1/1), same, near Rybachiy, bank of Kuma River, 20.VI.1974 1 ㅇ (CAP, \#40.18.1/3), same, near Rybachiy, VI.1974, all leg. A Ponomarev; 1 क (CAP, \#40.18.1/4), $23 \mathrm{~km}$ N of Kaspiyskiy, nea Djalykovo, from burrow of small ground squirrel (Spermophilus pygmaeus), 10.VII.1975, leg. V. Kuznetsova.

REMARKS. Mecynargus minutipalpis has recently been described from the Kherson Area and Crimea, both Ukraine. Previously, this species was erroneously referred to from the Kalmyk Republic, Russia as $M$. longus (Kulczyński, 1882) [Minoransky \& Ponomarev, 1984; Ponomarev \& Minoransky, 1984; Ponomarev, 1988]. The species is new to the Russian fauna.

\section{Bathyphantes similis Kulczyński, 1894}

1995 Bathyphantes similis. - Esyunin et al.: 53, misidentification, examined.

2007 B. similis. - Esyunin et al.: 46, misidentification, examined.

MATERIAL EXAMINED. Bathyphantes gracilis: 1 ( $\mathrm{PSU}$ \#18560, as B. similis), RUSSIA, Orenburg Area, near Sol'-Iletskiy, 20.VIII.2001, leg. S. Esyunin; 2 + (PSU, \#5833, as B. similis), Perm Area, Kungur Distr., near Erchach, meadow, IX.1968, leg. Panova; $2 \sigma^{7} \sigma^{7}, 8$ + 9 (PSU, \#1861, as B. similis), Perm Area, near Lake Dolgoye, swamp, 15.VIII.-21.IX.1991, leg. V. Kuzminykh; $2 \sigma^{7} \sigma^{7}, 3$ OO (PSU, \#4009, as B. similis), Tyumen' Area, YamaloNenets Autonomous Region, S of Yamal Peninsula, Khadyta-Yakha River flow, floodland meadow, 25.VI.1979, leg. S. Esyunin.

COMPARATIVE MATERIAL EXAMINED. Bathyphantes similis: 1 ○, 2 क् (MHNG), SUISSE, Vaud, Grotte du Ruisseau, VD 8, 3.XI.1984, leg. V. Aellen \& P. Strinati, det. K. Thaler; 3 of (MHNG), same, 3.XI.1984, leg. P. Strinati \& V. Aellen, det. K Thaler; $2 \sigma^{7} \sigma^{7}, 2$ 20 (MHNG), Grotte du Poits de Vaulion, VD, 31-7.10.1986, leg. P. Strinati, det. K. Thaler.

REMARKS. All Russian records of $B$. similis from the Urals [Esyunin et al., 1995] and Orenburg Area [Esyunin et al., 2007] are misidentifications and refer to $B$. gracilis. As a result, the species has not been reliably documented from Russia.

ACKNOWLEDGEMENTS. I am most grateful to all persons whose collections used in the present study. Thanks go to Alexander Ponomarev (Rostov-on-Don, Russia) and Donald Buckle (Saskatchewan, Canada) for providing material from their personal collections, Yuri Marusik (IBPN), Peter Jäger and Julia Altmann (SMF), Peter Schwendinger (MHNG), Torbjorn Kronestedt (NHRS), Dominika MierzwaSzymkowiak (MIZ), Kirill Mikhailov (ZMMU), Sergei Esyu- nin (PSU), Fabrizio Rigato (MCSNM) and Peter van Helsdingen (Leiden, The Netherlands) for the help in the receipt of type and/or other comparative material. Sergei Golovatch (Moscow, Russia) kindly checked the English of the final draft

\section{References}

Andreeva E.M., Tystshenko V.P. 1970. [Material to the spider fauna of Tajikistan] // Zool. Zh. T.49. No.1. P.38-44 [in Russian].

Andreeva E.M. 1976. [Spiders of Tadjikistan. The fauna and zonal-ecological distribution]. Dushanbe: Donish Publ. 193p. [in Russian]

Banks N. 1899. Arachnida // Ashmead W.H. (ed.). Reports upon the insects, mites and myriapods collected by Dr. L. Stejneger and Mr. G.E.H. Barrett-Hamilton on the Commander Islands. Report of Fur-Seal Investigations 1896-97. P.328351.

Benell-Aitchison C.W., Dondale C.D. 1990. A checklist of Manitoba spiders (Araneae) with notes on geographic relationships // Naturaliste Canadien. No.117. P.215-237.

Caporiacco L., di. 1935. Aracnidi dell'Himalaia e del Karakoram, raccolti dalla Missione italiana al Karakoram (1929-VII) // Mem. Soc. Entomol. Ital. T.13. P.161-263.

Emerton J.H. 1923. New spiders from Canada and the adjoining states, No. 3 // Canad. Ent. Vol.55. P.238-243.

Eskov K.Yu. 1986. [The spider fauna of the hypoarctic belt of Siberia] // Chernov Yu.I. (ed.). Yuzhnye tundry Taimyra. Leningrad: Nauka. P.174-191 [in Russian].

Eskov K.Yu. 1988. [Spiders (Aranei) of central Siberia] // Syroechkovsky E.E. (ed.). Materialy po faune Srednei Sibiri i prilezhashchikh raionov Mongolii. Moscow: TSNIL Glavokhoty. P.101-155 [in Russian].

Eskov K.Yu. 1992. [New data on the fauna of the spider family Linyphiidae (Aranei) of the Soviet Far East] // Ovtsharenko V.I. (ed.). Fauna i ekologiya paukov, skorpionov i lozhnoskorpionov SSSR. Trudy Zool. Inst. Akad. nauk SSSR, Leningrad. T.226. P.52-60 [in Russian].

Eskov K.Yu. 1992. New data on the linyphiid spider fauna of South Siberia (Aranei Linyphiidae) // Arthropoda Selecta. Vol.1. No. 2. P.73-82.

Eskov K.Yu. 1994. Catalogue of the linyphiid spiders of northern Asia (Arachnida, Araneae, Linyphiidae). Sofia-Moscow: Pensoft Publ. 144 pp.

Eskov K.Yu., Marusik Yu.M. 1994. New data on the taxonomy and faunistics of North Asian linyphiid spiders (Aranei Linyphiidae) // Arthropoda Selecta. Vol.2 (for 1993). No.4. P.41-79.

Esyunin S.L., Efimik V.E., Polyanin A.B. 1995. Remarks on the Ural spider fauna, 5. New records of spider species of the family Linyphiidae from the Urals (Arachnida Aranei) // Arthropoda selecta. Vol.4. No.2. P.49-71.

Esyunin S.L., Tuneva T.K., Farzalieva G.Sh. 2007. Remarks on the Ural spider fauna (Arachnida: Aranei). 12. Spiders of the steppe zone of Orenburg Region // Arthropoda Selecta. Vol.16. No.1. P.43-63.

Gnelitsa V.A. 2008. Pelecopsis steppensis sp.n. (Aranei: Linyphiidae) from southern Ukraine // Arthropoda Selecta. Vol.16. No.2. P.137-141 (for 2007).

Gnelitsa V.A. 2011. Mecynargus minutipalpis sp.n. (Araneae: Linyphiidae) from Ukraine // Acta Zool. Bulg. Vol.63. P.61-66.

Holm A. 1960. On a collection of spiders from Alaska // Zool. Bidr. Upps. Vol.33. P.109-134.

Holm Å. 1970. Notes on spiders collected by the "Vega" Expedition 1878-1880 // Entomologica Scand. Vol.1. P.188-208.

Keyserling E. 1886. Die Spinnen Amerikas. Theridiidae. Nürnberg. Bd.2. $295 \mathrm{~S}$.

Koponen S., Marusik Yu.M., Tanasevitch A.V. 1998. New data on the spider fauna of the Polar Urals (Aranei) // Arthropoda Selecta. Vol.6 (for 1997). No.3/4. P.109-119. 
Kryzhanovsky O.L. 2002. [Composition and distribution of entomofaunas of the globe]. Moscow KMK. 237 p. [in Russian].

Kulczyński W. 1885. Araneae in Camtschadalia a Dre B. Dybowski collectae // Pam. Wydz. matem.-przyr. Akad. Umiej. Kraków. T.11. P.1-60.

Logunov D.V., Marusik Yu.M., Koponen S. 1998. A check-list of the spiders in Tuva, South Siberia with analysis of their habitat distribution // Ber. nat.-med. Vereins Innsbruck. Bd.85. S.125-159.

Marusik Yu.M., Eskov K.Yu., Logunov D.V., Basarukin A.M. 1993. A check-list of spiders (Arachnida Aranei) from Sakhalin and Kurile Islands // Arthropoda Selecta. Vol.1 (for 1992). No.4. P.73-85.

Marusik Yu.M., Tanasevitch A.V., Kurenshchikov D.K., Logunov D.V. 2007. A check-list of the spiders (Araneae) of the Bolshekhekhtsyrski Nature Reserve, Khabarovsk Province, the Russian Far East // Acta Arachnol. Sinica. Vol.16. No.1. P.37-64.

Merrett P., Dawson I. 2005. Revalidation of Wabasso replicatus (Holm, 1950), and description from Britain (Araneae: Linyphiidae) // Bull. Br. arachnol. Soc. Vol.13. Pt.4. P.117-121.

Minoransky V.A., Ponomarev A.V. 1984. [Material to the spider fauna of Kalmykia] // Utochkin A.S. (ed.). Fauna i ekologiya paukoobrazhykh. Mezhvuzovskii sbornik nauchnykh trudov. Perm: Perm University. P.82-92 [in Russian].

Pickard-Cambridge O. 1873. On some new species of Araneidea, chiefly from Oriental Siberia // Proc. Zool. Soc. London. 1873 P. $435-452$, pl.XL-XLI.

Platnick N.I. 2013. The world spider catalog, version 13.5. American Museum of Natural History. Online at http://research.amnh. org/iz/spiders/catalog. DOI: 10.5531/db.iz.0001.

Polchaninova N.Yu. 2013. A check list of spiders (Araneae) of the Black Sea Biosphere Reserve (Ukraine) // Pryrodnychyi almanakh. Biolohichni nauky, vypusk 18. 2012. Zbirnyk naukovych pratz. Kherson: PAT "Khersonska miska drukarnia". P.85-108 [in Russian]

Ponomarev A.V. 1988. [Characteristics of the spider fauna of the semi-desert zone of the USSR European part.] // Fauna i ekologiya paukoobraznykh. Mezhvuzovskii sbornik nauchnykh trudov. Perm: Perm University. P.51-61 [in Russian].

Ponomarev A.V., Minoransky V.A. 1984. [A zoogeographic characteristic of Kalmykia's spiders] // A.G. Voronov (ed.). 8th Vses. zoog. konf. Leningrad, 6-8 Febr. 1985. Tez. dokl. Moscow: Zool. Inst. AN SSSR. P.233-235 [in Russian].

Schenkel E. 1930. Die Araneiden der schwedischen KamtchatkaExpedition 1920-1922 // Ark. Zool. Bd.21. H.15. S.1-33.

Starega W. 1974. Baldachinspinnen (Aranei: Linyphiidae) aus der Mongolei // Annales Zool. Warszawa. T.32. P.19-27.

Sytshevskaja V.I. 1935. Etude sur les Araignées de la Kamtchatka // Folia Zool. Hydrobiol. T.8. No.1. P.80-103.
Tanasevitch A.V. 1989. The linyphiid spiders of Middle Asia (Arachnida: Araneae: Linyphiidae) // Senckenbergiana Biol. Bd.69. H.1/3. S.83-176.

Tanasevitch A.V. 2005. Linyphiid spiders of the West Siberian Plain (Arachnida: Aranei) // Arthropoda Selecta. Vol.14. No.1. P.33-88.

Tanasevitch A.V. 2006. On some Linyphiidae of China, mainly from Taibai-Shan, Qinling Mountains, Shaanxi Province (Arachnida: Araneae) // Zootaxa. Vol.1325. P.277-311.

Tanasevitch A.V. 2007. On a small linyphiid spider collection from Simushir Island, Kurile Islands, Russia, with notes on Stemonyphantes sibiricus Grube (Aranei: Linyphiidae) // Arthropoda Selecta. Vol.15 (for 2006). No.3. P.255-258.

Tanasevitch A.V. 2008. New records of linyphiid spiders from Russia, with taxonomic and nomenclatural remarks (Aranei: Linyphiidae) // Arthropoda Selecta. Vol.16 (for 2007). No.2. P.115-135.

Tanasevitch A.V. 2010. On synonymy of linyphiid spiders of the Russian fauna (Arachnida: Aranei: Linyphiidae). 1 // Arthropoda Selecta. Vol.19. No.4. P.273-282.

Tanasevitch A.V. 2011. On synonymy of linyphiid spiders of the Russian fauna (Arachnida: Aranei: Linyphiidae). 2 // Arthropoda Selecta. Vol.20. No.2. P.129-143.

Tanasevitch A.V. 2012. A new Porrhomma Simon, 1884 from the mountains of southern Siberia, Russia (Aranei, Linyphiidae, Linyphiinae) // Arthropoda Selecta. Vol.21. No.4. P.369-374.

Tanasevitch A.V., Fet V.Y. 1986. [Material to the spider fauna (Aranei) of Turkmenistan. III. Family Linyphiidae] // Izv. Akad. nauk Turkmen. SSR, Biol. No.1. P.33-42 [in Russian].

Tanasevitch A.V., Koponen S. 2007. Spiders (Aranei) of the southern tundra in the Russian Plain // Arthropoda Selecta. Vol.15. No.4. P.295-345 (for 2006).

Tanasevitch A.V., Rybalov L.B. 2010. On spiders from the tundra zone of the Kola Peninsula, Russia (Arachnida: Aranei) // Arthropoda Selecta. Vol.19. No.1. P.41-56.

Tanasevitch A.V., Trilikauskas L.A. 2004. Check-list of the linyphiid spiders of the Bureinsky State Nature Reserve and adjacent territories, Russian Far East (Aranei: Linyphiidae) // Arthropoda Selecta. Vol.13. No.1-2. P.77-86.

Trilikauskas L.A., Tanasevitch A.V. 2006. New records of the linyphiid spiders from the Bureinsky Nature Reserve, Khabarovsk Province, Russian Far East // Arthropoda Selecta. Vol.14. No.4. P.339-345 (for 2005).

Tullgren A. 1955. Zur Kenntnis schwedischer Erigoniden // Ark. Zool. (N.S.). Bd.7. S.295-389.

Wunderlich J. 1995. Linyphiidae aus der Mongolei (Arachnida: Araneae) // Beitr. Araneol. Bd.4. S.479-529.

Xia Q., Zhang G.R., Gao J.C., Fei R., Kim J.P. 2001. Three new species of spiders of Erigoninae (Araneae: Linyphiidae) from China // Korean Arachnol. Vol.17. P.161-168.

Responsible editor K.G. Mikhailov 